\title{
Variable interstellar radiation fields in simulated dwarf galaxies: supernovae versus photoelectric heating
}

\author{
Chia-Yu Hu, ${ }^{1,2 \star}$ Thorsten Naab, ${ }^{1}$ Simon C. O. Glover, ${ }^{3}$ Stefanie Walch ${ }^{4}$ \\ and Paul C. Clark ${ }^{5}$ \\ ${ }^{1}$ Max-Planck-Institut für Astrophysik, Karl-Schwarzschild Strasse 1, D-85740 Garching, Germany \\ ${ }^{2}$ Center for Computational Astrophysics, Flatiron Institute, 162 th Ave, New York, NY 10010, USA \\ ${ }^{3}$ Zentrum für Astronomie der Universität Heidelberg, Institut für Theoretische Astrophysik, Albert-Ueberle-Str 2, D-69120 Heidelberg, Germany \\ ${ }^{4}$ Physikalisches Institut der Universität zu Köln, Zülpicher Strasse 77, D-50937 Köln, Germany \\ ${ }^{5}$ School of Physics and Astronomy, Cardiff University, 5 The Parade, Cardiff CF24 3AA, Wales, UK
}

Accepted 2017 July 12. Received 2017 June 24; in original form 2017 January 30

\begin{abstract}
We present high-resolution hydrodynamical simulations of isolated dwarf galaxies including self-gravity, non-equilibrium cooling and chemistry, interstellar radiation fields (ISRF) and shielding, star formation, and stellar feedback. This includes spatially and temporally varying photoelectric (PE) heating, photoionization, resolved supernova (SN) blast waves and metal enrichment. A new flexible method to sample the stellar initial mass function allows us to follow the contribution to the ISRF, the metal output and the SN delay times of individual massive stars. We find that SNe play the dominant role in regulating the global star formation rate, shaping the multiphase interstellar medium (ISM) and driving galactic outflows. Outflow rates (with mass-loading factors of a few) and hot gas fractions of the ISM increase with the number of SNe exploding in low-density environments where radiative energy losses are low. While PE heating alone can suppress star formation as efficiently as $\mathrm{SNe}$ alone can do, it is unable to drive outflows and reproduce the multiphase ISM that emerges naturally whenever $\mathrm{SNe}$ are included. We discuss the potential origins for the discrepancy between our results and another recent study that claimed that PE heating dominates over SNe. In the absence of SNe and photoionization (mechanisms to disperse dense clouds), the impact of PE heating is highly overestimated owing to the (unrealistic) proximity of dense gas to the radiation sources. This leads to a substantial boost of the infrared continuum emission from the UV-irradiated dust and a far-infrared line-to-continuum ratio too low compared to observations.
\end{abstract}

Key words: galaxies: dwarf-galaxies: ISM-galaxies: star formation.

\section{INTRODUCTION}

Stellar feedback is an indispensable ingredient in the theory of galaxy formation and evolution (Larson 1974; White \& Rees 1978; Dekel \& Silk 1986; Mac Low \& Ferrara 1999). Current large-scale cosmological simulations that incorporate efficient stellar feedback have been able to successfully reproduce many properties of observed galaxies (see reviews by Somerville \& Davé 2015 and Naab \& Ostriker 2017 and references therein). However, these simulations still lack the required resolutions to directly follow the feedback processes. As such, most of them have to resort to ad hoc sub-grid recipes in order to make the feedback efficient. These feedback recipes require explicit parameter calibration to certain observations and therefore should be viewed as a phenomenologi- cal 'input' of the model, which means that they are inadequate for investigating how stellar feedback couples with the gas dynamics on parsec scales. To do so, stellar feedback should be directly simulated on a scale that properly captures its dynamical impact on the interstellar medium (ISM) without fine tuning free parameters to match observations. This is, however, a very demanding task in terms of numerical resolution, and the trade-off one often has to make is to focus only on a single gas cloud or a small patch of a galaxy (see e.g. Joung \& Mac Low 2006; Dale \& Bonnell 2011; Kim, Kim \& Ostriker 2011; Hill et al. 2012; Walch et al. 2012, 2015; Simpson et al. 2016; Naab \& Ostriker 2017).

Dwarf galaxies, thanks to their relatively small sizes and low masses, provide a unique opportunity for simulators to conduct full-disc simulations at the required resolution (Kim et al. 2013a,b; Forbes et al. 2016; Hu et al. 2016). Moreover, they serve as ideal laboratories where numerical experiments can be conducted to gain insight into low-metallicity systems, which are very 
different from local spiral galaxies and so may provide important clues to the behaviour of high-redshift galaxies that also have low metallicity.

In a previous study (Hu et al. 2016, hereafter Paper I), we presented a set of detailed simulations of isolated dwarf galaxies. The model included gravity, hydrodynamics, non-equilibrium cooling and chemistry, radiation shielding, star formation, stellar feedback and metal enrichment. We found that the star formation rate (SFR) in the simulated galaxies was insensitive to the adopted (constant) strength of the interstellar radiation field (ISRF), because the supernova (SN) feedback dominates over the photoelectric (PE) heating (which depends on the ISRF) and therefore drives the ISM out of thermal equilibrium. These simulated galaxies have a long gas depletion time in agreement with the observed dwarf galaxies whenever SN feedback is included.

In this paper, we adopt a similar approach to the method used in Forbes et al. (2016) to compute the ISRF self-consistently, i.e. the ISRF is directly calculated from the stellar particles such that the ISRF varies both spatially and temporally. The dust extinction is assumed to be negligible, which should be a good approximation on the scales of interest in dust-poor dwarf galaxies. This allows us to calculate the ISRF without involving radiative transfer that is computationally expensive. We also improve our ISM model by introducing a new method of sampling stars from the stellar initial mass function (IMF) and assigning them to the star particles which allows us to form individual massive stars in the simulations. Finally, in addition to the feedback from PE heating and SNe that were already included in Paper I, we include feedback from photoionization using a Strömgren-volume approximation that can deal with overlapping $\mathrm{H}_{\mathrm{II}}$ regions. We investigate the impact of stellar feedback on the SFR and the galactic outflow rate in dwarf galaxies using different combinations of these feedback processes and examine their systematic differences. We come to the same conclusion as in Paper I (which is in conflict with Forbes et al. 2016) that SN feedback plays the dominant role in regulating star formation and shaping the ISM in dwarf galaxies, and we discuss the potential cause of this conflict in Section 4.

This paper is organized as follows: In Section 2, we describe the details of our numerical method and our setup of the initial conditions. In Section 3, we present the results of our numerical simulations. In Section 4, we discuss the implications of our results and the potential caveats. In Section 5, we summarize our work.

\section{NUMERICAL METHOD}

\subsection{Gravity and hydrodynamics}

We use the GADGET-3 code (Springel 2005), which solves the gravity with a tree-based method and solves hydrodynamics with the smoothed particle hydrodynamics (SPH) method (Gingold \& Monaghan 1977; Lucy 1977). We adopt the modified version of SPH described in $\mathrm{Hu}$ et al. (2014) which has improved accuracy and avoids some of the numerical artefacts that traditional SPH is prone to develop in multiphase flows. Briefly, we adopt the pressureenergy SPH formulation (Hopkins 2013; Saitoh \& Makino 2013), the Wendland $C^{4}$ kernel (Dehnen \& Aly 2012) with the number of neighbouring particles $N_{\text {ngb }}=100$ in the support radius and a switch for the artificial viscosity (Cullen \& Dehnen 2010) and artificial thermal conduction (Price 2008; Read, Hayfield \& Agertz 2010) devised to only operate at shocks. Detailed validation tests of this code are presented in $\mathrm{Hu}$ et al. (2014).

\subsection{Cooling and chemistry}

The model of cooling and chemistry we adopt follows Paper I, which itself is based on Nelson \& Langer (1997), Glover \& Mac Low (2007) and Glover \& Clark (2012a). We adopt a simplified chemistry network that traces seven chemical species: $\mathrm{H}_{2}, \mathrm{H}^{+}, \mathrm{CO}$, $\mathrm{H}, \mathrm{C}^{+}, \mathrm{O}$ and free electrons. The first three species are explicitly followed while the rest are calculated from the laws for element and charge conservation. Silicon is assumed to be always in the form of $\mathrm{Si}^{+}$(singly ionized). Carbon and oxygen, if not in the form of $\mathrm{CO}$ (carbon monoxide), are always in the form of $\mathrm{C}^{+}$and $\mathrm{O}$, respectively. Our hydrogen chemistry model assumes that $\mathrm{H}_{2}$ can only be formed through the 'dust channel', ${ }^{1}$ while it can be destroyed by photodissociation, collisional dissociation and cosmic ray (CR) ionization. The carbon chemistry assumes that $\mathrm{CO}$ formation is dictated by the process $\mathrm{C}^{+}+\mathrm{H}_{2} \rightarrow \mathrm{CH}_{2}^{+}$, and $\mathrm{CH}_{2}^{+}$will either react with $\mathrm{O}$ and form $\mathrm{CO}$ or be destroyed by photodissociation.

We follow non-equilibrium cooling and heating processes as described in Paper I, with a major difference that in this work we no longer assume a uniform constant ISRF. Instead, we calculate the ISRF directly from the stellar particles and therefore make the ISRF spatially and temporally variable (see Section 2.4). We apply our non-equilibrium cooling model only if the gas temperature $T$ is lower than $T_{\mathrm{c}}=3 \times 10^{4} \mathrm{~K}$. When $T>T_{\mathrm{c}}$, we use an equilibrium cooling table from Wiersma, Schaye \& Smith (2009) instead, ${ }^{2}$ which depends on 11 different elements (including hydrogen and helium) that will evolve due to metal enrichment (see Section 2.5.3). The transition of the cooling rate is smooth if the hydrogen is in collisional ionization equilibrium in the low-temperature regime where we account for non-equilibrium effects, which is not generally true. This leads to a small discontinuity in the cooling rate around $T_{\mathrm{c}}$ (which manifests itself as a thin line in Figs 9 and 11). However, this (artificial) discontinuity has only a minor effect on the dynamical behaviour of the ISM (more specifically, the evolution of the $\mathrm{SN}$ remnants) as long as cooling is rapid in this temperature range. We have conducted idealized tests using such a method in Paper I and found a good agreement with analytic estimates. In this work we deliberately turn off CR ionization even though it is included in our ISM model, in order to focus on the effect of the three feedback mechanisms (described in Section 2.5) and to aid comparison with Forbes et al. (2016).

The local dust temperature is calculated following the method of Glover \& Clark (2012a), assuming that the dust is in thermal equilibrium where radiative cooling of dust balances dust heating due to absorption of the ISRF, $\mathrm{H}_{2}$ formation on dust and dust-gas collisions. Under the conditions, our simulations explore in this work, dust heating due to the ISRF is the dominant process.

\subsection{Star formation}

\subsubsection{The star formation recipe}

Star formation is a multiscale process. Following the entire dynamical range relevant for star formation is beyond current computational capability, and therefore one always has to resort to sub-resolution

\footnotetext{
${ }^{1}$ Even at the relatively low metallicities considered in this study, this remains a good approximation; see e.g. the discussion in Glover (2003).

${ }^{2}$ We adopt the collisional ionization equilibrium version of the table from Wiersma et al. (2009) as we assume the ISM is self-shielded against the cosmic UV background, which should be a good approximation given the column density of the discs.
} 
models. The star formation recipes generally fall into two different categories: the 'sink particle' approach (Bate, Bonnell \& Price 1995; Li, Mac Low \& Klessen 2005; Federrath et al. 2010; Hubber, Walch \& Whitworth 2013; Gatto et al. 2017) often used in the small-scale (parsec or sub-parsec) simulations and the 'Schmidt-type' stochastic approach (Schmidt 1959; Cen \& Ostriker 1992; Katz 1992) often used in large-scale $(0.1 \mathrm{kpc}$ or larger) cosmological simulations. As our resolution falls between these two scales, the choice is not obvious and it deserves some discussion as follows.

In small-scale simulations, sink particles serve as an efficient device to remove unresolved dense gas from the simulations. However, when the resolution is poor, not all of the unresolved gas is going to form stars eventually. A large fraction of the gas would thus be artificially locked up into sink particles, if the conventional Jeans criteria for forming sink particles is adopted (Bate \& Burkert 1997; Truelove et al. 1997). Although the issue could be alleviated by adopting a sub-grid model to account for the gas artificially locked up in sink particles, this gas would never be released back to the ISM, which happens in a feedback-regulated system. Another disadvantage of this approach is that sink particles are usually much more massive than the gas particles and therefore the stellar gravity is less well resolved compared to the gas. We therefore refrain from using the sink particle approach.

Instead, we choose to adopt the 'Schmidt-type' stochastic approach as

$\dot{\rho}_{*}=\epsilon_{\mathrm{sf}} \frac{\rho_{\mathrm{gas}}}{t_{\mathrm{ff}}} \propto \rho_{\mathrm{gas}}^{1.5}$,

where $\dot{\rho}_{*}$ is the instantaneous SFR density, $\rho_{\text {gas }}$ is the gas density, $t_{\mathrm{ff}}$ is the gas free-fall time and $\epsilon_{\mathrm{sf}}$ is an efficiency parameter which we assume to be 0.02 . Unlike the sink particle approach, an unresolved gas particle in this recipe would not be locked up into stars instantaneously. Instead, it is converted to a star particle with the same mass stochastically on a time-scale of $t_{\mathrm{ff}} / \epsilon_{\mathrm{sf}}$. Therefore, the unresolved gas has a chance to be dispersed into the diffuse phase again before it forms stars.

\subsubsection{The star formation threshold}

Unlike in Paper I, where we adopted both density and temperature thresholds for star formation, in this work we adopt a Jeans star formation threshold which only involves one free parameter. For every gas particle, its associated Jeans mass is defined as

$M_{\mathrm{J}, i}=\frac{\pi^{5 / 2} c_{s, i}^{3}}{6 G^{3 / 2} \rho_{i}^{1 / 2}}$,

where $G$ is the gravitational constant, and $c_{s, i}$ and $\rho_{i}$ are the sound speed and the density of the gas particle $i$, respectively. A gas particle $i$ will be assigned with a non-zero instantaneous SFR and will be 'star-forming' only if $M_{\mathrm{J}, i}<N_{\mathrm{th}} M_{\mathrm{ker}}$, where $M_{\mathrm{ker}}=N_{\mathrm{ngb}} m_{\mathrm{gas}}$ is the SPH kernel mass and $N_{\text {th }}$ is a free parameter. In this work, we choose $N_{\text {th }}=8$ in order to properly resolve the Jeans mass for the star-forming gas. The effect of varying $N_{\text {th }}$ is investigated in Appendix B1. In addition, we further require that the gas has to have a negative local velocity divergence (i.e. converging flows) in order to be star-forming.

\subsubsection{Sampling the stellar masses from an IMF}

In large-scale cosmological simulations where the resolutions are limited ( $m_{\text {gas }} \gtrsim 10^{5} \mathrm{M}_{\odot}$ ), a star particle usually represents a cluster of stars. Adopting a simple stellar population model with an assumed IMF, one can determine the fraction of massive stars and calculate the energy budget of the stellar feedback for each star particle. For a Kroupa IMF (Kroupa 2001), for example, there is around one type II SN per $100 \mathrm{M}_{\odot}$ of stars formed. A star particle of mass $m_{*}$ would therefore inject $\left(m_{*} / 100 \mathrm{M}_{\odot}\right) E_{51}$ of energy into the ISM, where $E_{51}=10^{51} \mathrm{erg}$ is the canonical energy released by a single SN event (see Janka 2012).

However, as the mass of a star particle becomes smaller than $100 \mathrm{M}_{\odot}$, the natural minimal unit of $\mathrm{SNe}$ is reached and it is unphysical to still inject $\left(m_{*} / 100 \mathrm{M}_{\odot}\right) E_{51}$ into the ISM for each star particle. In addition, if $\left(m_{*} / 100 \mathrm{M}_{\odot}\right) E_{51}$ were injected into the ambient gas with $m_{\text {gas }}=m_{*}-$ the approach usually adopted in the literature - then the mass resolution of the hot gas would be too poor to properly resolve the Sedov-Taylor phase of the SN remnant, leading to serious numerical overcooling. This motivates the stochastic feedback injection approach that is adopted in Dalla Vecchia \& Schaye (2012) and Hu et al. (2016), where each star particle will have a probability of $1 / N_{\mathrm{c}}\left(N_{\mathrm{c}}>1\right)$ to inject a feedback energy of $\left(N_{\mathrm{c}} m_{*} / 100 \mathrm{M}_{\odot}\right) E_{51}$ into the ISM at the end of its lifetime. Here $N_{\mathrm{c}}$ is an arbitrary clustering factor that can be chosen as $100 \mathrm{M}_{\odot} / m_{*}$ to make sure that each injection represents an SN event. One can even adopt $N_{\mathrm{c}}>100 \mathrm{M}_{\odot} / m_{*}$, which physically means that $N_{\mathrm{c}} m_{*} / 100 \mathrm{M}_{\odot} \mathrm{SN}$ events are clustered together both spatially and temporally, in order to better resolve the Sedov-Taylor phase of the SN remnant (at the expense of poorer sampling of SN events due to clustering). However, in the stochastic approach the mass injection and the metal enrichment cannot be done consistently with the energy injection, simply because a star particle may have less mass and metals than the $\mathrm{SN}$ ejecta.

Another caveat of this approach is that it implicitly assumes that the IMF is always uniformly sampled within a star particle, which is obviously inappropriate when the mass of a star particle becomes smaller than $100 \mathrm{M}_{\odot}$. This may not be an issue when the SFR is high and a large number of stars are formed locally. However, when the local SFR is very low, which is common in dwarf galaxies, the sampling fluctuations of the IMF can be significant (see da Silva, Fumagalli \& Krumholz 2012, 2014 for the effects of the sampling fluctuations).

In this work, instead of interpreting the star particle as a simple stellar population that fairly samples the IMF, we introduce a flexible method to sample an IMF where each star particle represents an individual star when the resolution is high, while in the low resolution limit the simple stellar population model is recovered. The method is most useful when the mass resolution is close to $\mathrm{M}_{\odot}$, where the sink particle approach suffers from resolution issues and the simple stellar population does not apply.

In this method, a gas particle is first converted to a star particle of the same mass using the stochastic star formation recipe. Then, for each star particle of mass $m_{*}\left(m_{*}=m_{\mathrm{gas}}\right)$, we randomly draw an array of stellar masses $\left(m_{\mathrm{IMF}}\right)$ from the assumed IMF and assign them to the star particle until the total sampled stellar mass $M_{\mathrm{IMF}}=\sum m_{\mathrm{IMF}}$ equals or exceeds $m_{*}$. In practice, $M_{\mathrm{IMF}}$ is unlikely to exactly equal $m_{*}$, and there will always be a residual mass $M_{\mathrm{IMF}}-m_{*}$, which we transfer to the next star particle, so that the total mass is conserved. ${ }^{3}$ After the sampling is done, we modify the mass of the star particle to be $M_{\mathrm{IMF}}$. When the star particles are massive (poor resolution), the residual mass will only be a small fraction of the original $m_{*}$, so the star particles will still have almost

\footnotetext{
${ }^{3}$ Note that we cannot simply require that $M_{\mathrm{IMF}}=m_{*}$ as an additional constraint during the sampling, as this leads to us oversampling low-mass stars and undersampling high-mass stars (Sormani et al. 2017).
} 
equal mass after the sampling. On the other hand, when the star particles have masses of the order of a solar mass, even one massive $\mathrm{O}$ star can greatly exceed $m_{*}$. In this case, the subsequent star particles will be assigned with zero mass until the residual mass is exhausted. Star particles that are assigned with zero mass will be removed from the simulations. We note that modifying the mass implies an unphysical mass transfer between star particles, so some sort of distance constraint for the mass transfer is desirable, though it is not yet implemented in this work.

We adopt the Kroupa IMF and set the maximum and minimum masses to be 50 and $0.08 \mathrm{M}_{\odot}$, respectively. To sample random numbers following the Kroupa IMF, we use the acceptance-rejection method with a power-law envelope function, which has a high acceptance rate of about 65 percent. For the Kroupa IMF and our adopted mass range, the average number of stars per solar mass is 1.819 , which means that the average number of stars assigned in each star particle would be $1.819 \mathrm{~m}_{*} / \mathrm{M}_{\odot}$ with certain fluctuations. However, since stars below $1 \mathrm{M}_{\odot}$ do not contribute much to both the UV luminosity and the metal enrichment, we do not store them into the stellar mass array in order to save computer memory (but we still add them to $M_{\mathrm{IMF}}$ ). The average number of stars above $1 \mathrm{M}_{\odot}$ is $0.175 m_{*} / \mathrm{M}_{\odot}$, which is about 10 times less memory demanding.

\subsection{Interstellar UV radiation field}

We adopt an approximation similar to Forbes et al. (2016) which is only valid in dust-poor systems. In galaxies with a dust-to-gas ratio (DGR) close to that of the Milky Way, calculating a local ISRF is non-trivial because of dust extinction, and one usually has to resort to radiative transfer which is very computationally expensive. However, in dwarf galaxies where the DGR is significantly lower, dust extinction in the diffuse ISM becomes negligible and one can simply calculate the local ISRF by summing up all contributions from the stellar sources using the inverse square law.

For a given sampled stellar mass, we obtain the effective temperature and the stellar lifetime using the results of Georgy et al. (2013), which assume a metallicity $Z=0.002\left(\sim 0.1 \mathrm{Z}_{\odot}\right)$. We then adopt the stellar spectra from the Basel Stellar Library stellar library (Lejeune, Cuisinier \& Buser 1997, 1998; Westera et al. 2002) and integrate the spectra to obtain the UV luminosity in the energy range of $6-13.6 \mathrm{eV}$, which is relevant for the PE heating in the ISM. The total UV luminosity of a star particle is then obtained by summing over the contributions from all the assigned stellar masses whose ages are younger than their stellar lifetimes.

The energy density at the location of a gas particle is calculated by

$u_{6-13.6 \mathrm{eV}}=\sum_{i} \frac{L_{i, 6-13.6 \mathrm{eV}}}{4 \pi c r_{i}^{2}}$,

where $c$ is the speed of light, $L_{i, 6-13.6 \mathrm{eV}}$ is the UV luminosity of star particle $i$ and $r_{i}$ is the distance between the gas particle and the star particle. ${ }^{4}$ We take advantage of the gravity tree and store the UV luminosity into tree nodes. This allows us to perform the summation in equation (3) while walking the tree, which greatly reduces the computational cost. The tree approach gives exact results in the

\footnotetext{
${ }^{4}$ A star particle $i$ may contain several stars (see Section 2.3.3) and we sum

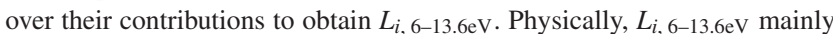
comes from stars with $m_{\mathrm{IMF}}>4 \mathrm{M}_{\odot}$ which traces the recent star formation on the time-scale of $100 \mathrm{Myr}$, though in practice we include all stars with $m_{\mathrm{IMF}}>1 \mathrm{M}_{\odot}$.
}

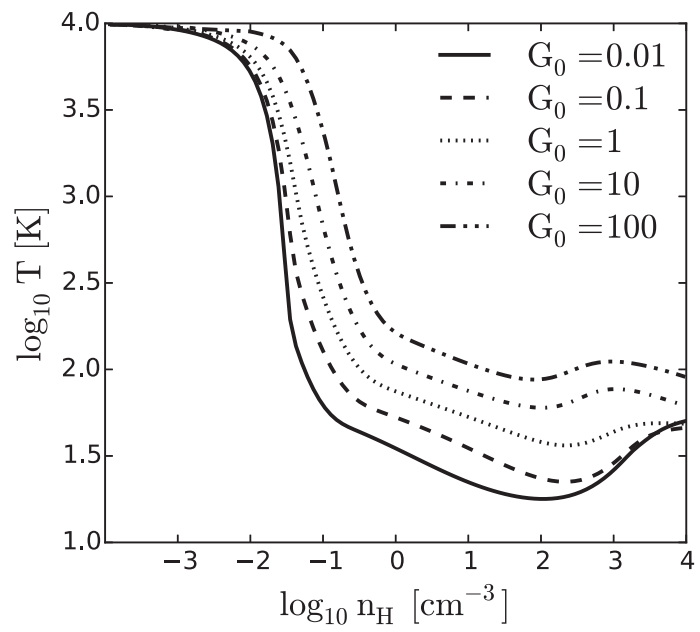

Figure 1. Temperature versus hydrogen number density for gas in thermal equilibrium under five different values of $G_{0}$, The adopted metallicity is $0.1 Z_{\odot}$ and the dust-to-gas mass ratio is 0.1 percent. Heating from CR ionization is deliberately switched off for simplicity and to aid comparison with Forbes et al. (2016). Increasing $G_{0}$ leads to a higher equilibrium temperature due to more efficient $\mathrm{PE}$ heating. In contrast to photoionization, which can heat dense gas up to $\sim 10^{4} \mathrm{~K}$, PE heating only heats dense gas up to a few hundred Kelvin even in the most extreme case.

vicinity of the star and starts to show some small deviation (controlled by the opening criteria of the gravity tree) at larger distances due to the tree approximation, which is sufficient for our purpose.

\subsection{Stellar feedback}

\subsubsection{Photoelectric heating}

As in Paper I, we adopt the following PE heating rate (Bakes \& Tielens 1994; Wolfire et al. 2003; Bergin et al. 2004):

$\Gamma_{\mathrm{pe}}=1.3 \times 10^{-24} \epsilon D G_{\mathrm{eff}} n \mathrm{erg} \mathrm{s}^{-1} \mathrm{~cm}^{-3}$,

where $G_{\text {eff }}=G_{0} \exp \left(-1.33 \times 10^{-21} D N_{\mathrm{H}, \mathrm{tot}}\right)$ is the attenuated radiation field strength in units of the Habing (1968) field, $n$ is the hydrogen nuclei number density and $\epsilon$ is the PE heating efficiency defined as

$\epsilon=\frac{0.049}{1+\left(0.004 \psi^{0.73}\right)}+\frac{0.037(T / 10000)^{0.7}}{1+2 \times 10^{-4} \psi}$,

where $\psi=G_{\text {eff }} T^{0.5} / n_{\mathrm{e}^{-}}$and $n_{\mathrm{e}^{-}}$is the electron number density. The major difference is that in this work $G_{0}$ is locally obtained by

$G_{0}=\frac{u_{6-13.6 \mathrm{eV}}}{5.29 \times 10^{-14} \mathrm{erg} \mathrm{cm}^{-3}}$,

where $u_{6-13.6 \mathrm{ev}}$ is calculated via equation (3), which varies both spatially and temporally. In the solar neighbourhood, the estimated $G_{0}$ is 1.7 (Draine 1978). We set a minimum value of $G_{0} \geq$ $G_{0, \min }=0.00324$, which is the cosmic UV background from Haardt \& Madau (2012) integrated over the 6-13.6 eV energy range.

Fig. 1 shows the gas (thermal-)equilibrium temperature $(T)$ as a function of its hydrogen number density $\left(n_{\mathrm{H}}\right)$ for several different strengths of the ISRF, assuming that the gas is optically thin. Increasing the ISRF leads to a larger PE heating rate and therefore a higher equilibrium temperature. As mentioned in Section 2.2, the CR ionization heating is not included throughout this work for simplicity and to aid comparison with Forbes et al. (2016). In the range of $n_{\mathrm{H}}=0.01-0.1 \mathrm{~cm}^{-3}$, the equilibrium curves have a steep 
negative slope where $T$ drops sharply as $n_{\mathrm{H}}$ increases. In the 'cold phase' regime $\left(n_{\mathrm{H}} \gtrsim 1 \mathrm{~cm}^{-3}\right)$, the scaling between $G_{0}$ and $T$ is sublinear: increasing $G_{0}$ by four orders of magnitude only leads to less than one order of magnitude increase in equilibrium temperature.

There can still be non-vanishing amount of radiation shielding especially in dense regions, and we treat it in the same way as Paper I, adopting the TREECOL algorithm (Clark, Glover \& Klessen 2012) to estimate the column densities along 12 different lines of sight for each gas particle. We calculate the unattenuated ISRF via equation (3) which includes contributions from all sightlines. This ISRF is then attenuated exponentially along each sightline based on its column density. The column densities are accumulated by taking advantage of the gravity tree structure up to a predefined shielding length $L_{\mathrm{sh}}=50 \mathrm{pc}$, assuming that most shielding comes from the nearby gas. In practice, we found that the shielding does not have a significant effect on the final SFR. ${ }^{5}$

\subsubsection{Photoionization}

Massive stars emit UV radiation that ionizes and heats up the ambient medium. This can lead to gas expansion in the immediate vicinity of the stars. As a result, when they explode as SNe, they do so in a low-density environment, rather than a dense molecular cloud. It is therefore important to account for photoionization feedback if we want to properly capture the effects of the $\mathrm{SNe}$ (see e.g. Peters et al. 2017).

Here we adopt a Strömgren type approximation for the photoionization similar to Hopkins, Quataert \& Murray (2012). For a given star particle with at least one assigned stellar mass $m_{\mathrm{IMF}}>8 \mathrm{M}_{\odot}$, we flag its neighbouring gas particles within a certain radius $R_{\mathrm{S}}$ as 'photoionized' and set their ionization fraction to be 1 . These gas particles are not allowed to cool below a minimum temperature of $10^{4} \mathrm{~K}$, the typical temperature in an $\mathrm{H}_{\text {II }}$ region.

In a medium of uniform density, $R_{\mathrm{S}}$ can be determined using the classical Strömgren radius $R_{\mathrm{s}}=\left(3 S_{*} / 4 \pi n^{2} \beta\right)^{1 / 3}$, where $S_{*}$ is the ionizing photon rate of the star, $\beta=2.56 \times 10^{-13} \mathrm{~cm}^{3} \mathrm{~s}^{-1}$ is the case B hydrogen recombination coefficient, evaluated at $T=10^{4} \mathrm{~K}$, and $n=\rho_{\mathrm{g}} X_{\mathrm{H}} / m_{\mathrm{p}}$ is the hydrogen nuclei number density where $X_{\mathrm{H}}$ is the hydrogen mass faction and $m_{\mathrm{p}}$ is the proton mass. However, if the density is inhomogeneous, the gas density estimated at the location of the star may be very different from the density of the neighbouring gas relevant for recombination. Moreover, when two Strömgren spheres overlap (e.g. two stars at the same location), this simple method would double-count the photoionized gas and therefore underestimate $R_{\mathrm{S}}$. As such, we determine $R_{\mathrm{s}}$ iteratively as follows.

Assuming ionization equilibrium, the hydrogen recombination of the neighbouring gas should balance the ionizing photon budget of the star. Each neighbouring gas particle contributes $\beta n_{\mathrm{H}} N_{\mathrm{H}}$ recombinations per second, where $N_{\mathrm{H}}=m_{\mathrm{g}} X_{\mathrm{H}} / m_{\mathrm{p}}$ is the number of hydrogen nuclei for the gas particle and $m_{\mathrm{g}}$ is the gas particle mass. For each star particle, we first search for a few $(\sim 10)$ neighbouring gas particles and calculate the total recombination rate $R_{\text {rec }}=\beta \sum_{i}\left(n_{\mathrm{H}}^{i} N_{\mathrm{H}}^{i}\right)$, where the summation is over the neighbouring particles $i$. To cope with overlapping $\mathrm{H}$ II regions, if a neighbouring gas particle is already flagged as 'photoionized' by another star particle, it will be excluded from the summation. We note that this process depends on the order of flagging for different star particles

\footnotetext{
${ }^{5}$ In Paper I where we adopted a constant ISRF, we showed that the results
} were rather insensitive to the choice of $L_{\mathrm{sh}}$. which may affect the reproducibility of the results, ${ }^{6}$ though statistically this is not a major concern. If $S_{*}-R_{\text {rec }}>0$, we look for more neighbours by increasing the searching radius by 10 percent and repeat the flagging, which is analogous to propagating the ionization front. If $S_{*}-R_{\text {rec }}<0$, we decrease the searching radius by 10 per cent and unflag the particles that are now outside of the radius. We do so iteratively until $\left|S_{*}-R_{\text {rec }}\right|$ is smaller than some tolerance, which is set to be $10 \beta m_{\mathrm{g}, \text { in }} X_{\mathrm{H}} / m_{\mathrm{p}}$. This tolerance is chosen to be resolution dependent and it corresponds to the recombination rate of one gas particle in an ambient hydrogen density of $10 \mathrm{~cm}^{-3}$. In addition, if the current iteration only flags/unflags one more gas particle compared to the previous iteration and $S_{*}-R_{\text {rec }}$ changes sign, we terminate the iteration as it means that this gas particle has a density that is too high such that the stopping criterion will never be met: performing more iterations would only repeatedly flag and unflag this gas particle. In Appendix A, we show that this approach converges and agrees well with that predicted by more sophisticated radiative transfer methods in a uniform medium, though in an inhomogeneous medium the method is expected to be strongly mass-biased.

Although our approach is more accurate than using the simple Strömgren radius as described above, it is still strongly mass-biased, i.e. it preferentially ionizes mass concentrated regions. This is because our approach ignores any angular information. For example, consider the case of a dense molecular cloud located at some distance from an ionizing source that is otherwise surrounded only by diffuse gas. The Lagrangian nature of SPH means that in this situation, the majority of the SPH particles in the vicinity of the ionizing source may be contained within the dense cloud. In our method, the cloud would therefore be assigned the majority of the ionizing flux, even though from the point of view of the ionizing source it may subtend only a small solid angle. One potential improvement in a future version of this method would be to include at least some angular information by subdividing the $4 \pi$ solid angle around each ionizing source into several pixels and applying the method separately to each pixel. For the time being, however, we prevent the method from artificially ionizing a dense cloud far away from the star by imposing a maximum $R_{\mathrm{S}}$ of $50 \mathrm{pc}$. This (purely empirical) value is motivated by the typical size of $\mathrm{H}_{\mathrm{II}}$ regions deduced from observations of nearby dwarf galaxies (see e.g. Cormier et al. 2015). Despite the approximate nature of this method, it captures the physical behaviour of photoionization at a low computational cost compared to the radiative transfer approach (see e.g. Rosdahl et al. 2015 and Peters et al. 2017).

\subsubsection{Supernovae and metal enrichment}

A star particle with at least one assigned stellar mass $m_{\mathrm{IMF}}>8 \mathrm{M}_{\odot}$ will explode as an SN type II (SNII) at the end of its lifetime obtained from Georgy et al. (2013). We further restrict the timesteps of massive star particles to a maximum of $0.1 \mathrm{Myr}$, in order to make sure that they explode promptly after the end of their lifetimes. In practice, however, the typical timesteps of stars are usually smaller than 0.1 Myr due to other timestep constraints (e.g. the gravitational acceleration). Regardless of the actual stellar mass, each SNII injects $10^{51} \mathrm{erg}$ of thermal energy into its nearest 100 particles weighted

\footnotetext{
${ }^{6}$ Our neighbour search is done globally instead of only on the local domain, which prevents artificial cut-off by domain decomposition.
} 
by a cubic spline kernel function. ${ }^{7}$ We do not include type Ia SNe in this work.

We adopt the metal yields of SNII from Chieffi \& Limongi (2004) to account for the metal enrichment. The mass of the total ejecta and individual elements are added to the nearest 100 gas particles, also weighted by the cubic spline kernel. Since the average mass of the gas particle can be much smaller than the ejecta mass of massive stars, the neighbouring gas particles can be much more massive than the rest of the gas particles after the enrichment, which will lead to undesirable numerical noise in SPH. Therefore, we split a gas particle into two particles whenever its mass becomes larger than $2 m_{\text {gas,init }}$, where $m_{\text {gas,init }}$ is the gas particle mass in the initial conditions, and we do so iteratively until all gas particles have their masses smaller than $2 m_{\text {gas,init }}$ to make sure that this is the maximum mass of gas particles at all times. The two split particles inherit all physical quantities from their parent particle (including the velocity, specific thermal energy, metallicity and chemical abundances) except for their mass, which is half the parent particle mass, and their positions, which are offset symmetrically by one-fifth of the smoothing length of the parent particle in a random direction.

\subsection{Initial conditions}

We set up the initial conditions using the code developed by Springel, Di Matteo \& Hernquist (2005). The dark matter halo has a virial radius $R_{\mathrm{vir}}=44 \mathrm{kpc}$ and a virial mass $M_{\mathrm{vir}}=2 \times 10^{10} \mathrm{M}_{\odot}$. It follows a Hernquist profile with an NFW-equivalent (Navarro, Frenk \& White 1997) concentration parameter $c=10$ and the spin parameter $\lambda=0.03$. A pre-existing stellar disc of $2 \times 10^{7} \mathrm{M}_{\odot}$ and a gas disc of $4 \times 10^{7} \mathrm{M}_{\odot}$ are embedded in the dark matter halo, which results in a low baryonic mass fraction of 0.3 percent that is motivated by abundance matching (Moster et al. 2010; Moster, Naab \& White 2013). The stellar disc follows an exponential profile with scalelength $l_{\text {star }}=0.73 \mathrm{kpc}$ and scaleheight of $0.35 \mathrm{kpc}$. The gas disc also follows an exponential profile, and in this work we explore two different initial conditions that differ only in their gas disc scalelengths $l_{\text {gas }}$ : one with $0.73 \mathrm{kpc}$ and the other with $1.46 \mathrm{kpc}$. The latter will be our fiducial choice as the gas disc is usually observed to be more extended than the stellar disc (Begum et al. 2008; Hunter et al. 2012). The difference in $l_{\text {gas }}$ leads to a factor of 3-4 difference in gas surface density in the central region of the disc. The scaleheight of the gas disc is determined assuming vertical hydrostatic equilibrium.

The initial gas temperature is set to be $10^{4} \mathrm{~K}$. The initial metallicity (both gas and stars) is set to be $0.1 \mathrm{Z}_{\odot}$ uniformly, and the relative abundances of the 11 elements follow the solar abundances. The dust-to-gas mass ratio is set to be 0.1 percent. We adopt a particle mass of $m_{\mathrm{dm}}=10^{4} \mathrm{M}_{\odot}$ for the dark matter, $m_{\text {disc }}=4 \mathrm{M}_{\odot}$ for the stellar disc, and $m_{\text {gas }}=4 \mathrm{M}_{\odot}$ for the gas. The gravitational softening length (Plummer-equivalent) is $62 \mathrm{pc}$ for the dark matter and $2 \mathrm{pc}$ for the baryons. In Appendix B2, we demonstrate that adopting a smaller gravitational softening length for the gas does not significantly affect the outcome of our simulations.

We use cylindrical coordinates $R$ and $z$ to describe the simulations, where $R$ is the galactocentric radius and $z$ is along the rotation axis of the disc. The origin is chosen at the centre of mass of the stellar disc. We define the 'ISM region' as $R<1.5 \mathrm{kpc}$ and $|z|<1 \mathrm{kpc}$,

\footnotetext{
${ }^{7}$ For the energy injection, the adopted kernel function has little effect on the results as opposed to the SPH interactions where the cubic spline kernel function suffers from the numerical clumping instability.
}

where the star formation takes place. The simulation time is denoted as $t$.

\subsubsection{Initial turbulent driving}

With the smooth initial conditions described above, we have seen that the gas quickly collapses along the $z$-axis to form a very thin layer, which in turn leads to a subsequent burst of star formation (for around $0.1 \mathrm{Gyr}$ or so) that creates a hole in the central part of the galaxy (see Paper I). We consider this a numerical artefact that motivated us to introduce pre-existing turbulence in our gas disc by running a setup simulation for $20 \mathrm{Myr}$. In the setup simulation, we switch off star formation and stellar feedback, but we inject thermal energy into the gas disc in a way that mimics the effect of SNe: we first identify locations of overdense gas particles (defined as $n_{\text {gas }}>0.1 \mathrm{~cm}^{-3}$ and $T>10^{4} \mathrm{~K}$ ) within the central region $R<1 \mathrm{kpc}$ and then stochastically inject $10^{51} \mathrm{erg}$ at each of these locations and its associated 100 neighbouring particles. The probability to inject this thermal energy for each overdense gas particle within a timestep of $\Delta t$ is $0.02 \Delta t / t_{\mathrm{ff}}$, in the same spirit as our stochastic star formation model. By doing so we obtain a turbulent gas disc with pre-existing structures. We found it to be an efficient way to avoid the initial burst of star formation and outflows, allowing the system to settle into a steady state more rapidly.

\subsubsection{Naming conventions}

We run 13 simulations with different combinations of stellar feedback, stellar lifetimes and initial conditions. The naming convention is listed in Table 1, where PE, PI and $S N$ stands for PE heating, photoionization and $\mathrm{SNe}$ (type II), respectively. The PE heating is switched off by setting $G_{0}=0$ (instead of $G_{0}=G_{0, \min }$ ) everywhere. We also run a simulation with a constant $G_{0}=0.1$ (denoted as $c P E$ ). The value is chosen to be linearly scaled with the SFR surface density, which in our fiducial simulation is roughly an order of magnitude lower than in the local ISM. ${ }^{8}$ Finally, we run a simulation where all the massive stars $\left(m_{\mathrm{IMF}}>8 \mathrm{M}_{\odot}\right)$ have a constant stellar lifetime of $3 \mathrm{Myr}$ (denoted as $3 M y r$ ). The initial condition with $l_{\text {gas }}$ $=0.73 \mathrm{kpc}$ will be referred to as the 'compact disc' and denoted as $c m p$ while the one with $l_{\text {gas }}=1.46 \mathrm{kpc}$ will be referred to as the 'fiducial disc'.

\section{RESULTS}

\subsection{The interstellar radiation field}

The left-hand panel of Fig. 2 shows a face-on map of the (massweighted) ISRF as seen by the gas at $z=0$ for the fiducial run $P E-P I-S N$ at $t=600 \mathrm{Myr}$. The star particles with $m_{*}>8$ and $4 \mathrm{M}_{\odot}<m_{*} \leq 8 \mathrm{M}_{\odot}$ are overplotted with blue and white circles, respectively. Their sizes are linearly proportional to their masses. Stars less massive than $4 \mathrm{M}_{\odot}$ do not contribute much to the ISRF and hence are not plotted. Note that since we adopt $m_{\text {gas }}=4 \mathrm{M}_{\odot}$ these star particles represent individual stars.

A smooth background radiation field that gradually declines with $R$ is naturally established from the UV-emitting star particles. In addition to the smooth background, the ISRF can be locally enhanced significantly in the vicinity of UV-emitting stars. This is especially visible for massive stars $\left(m_{*}>8 \mathrm{M}_{\odot}\right)$ which have higher luminosities, though they are very few in number both because of the IMF

\footnotetext{
${ }^{8}$ Recall that $G_{0} \sim 1$ in the solar neighbourhood.
} 
Table 1. Simulation runs and corresponding physics. Feedback processes that are included in the simulation runs are marked as 'yes', and 'no' otherwise. $l_{\text {gas }}$ and $l_{\text {star }}$ are the scalelengths of the gaseous disc and the pre-existing stellar disc, respectively, where $l_{\text {star }}$ $=0.73 \mathrm{kpc}$ in all runs. $c P E-P I-S N$ has a constant radiation field with $G_{0}=0.1$ instead of a spatially and temporally varying ISRF. $P E-P I-S N-3 M y r$ has a constant stellar lifetime of $3 \mathrm{Myr}$ for all massive stars.

\begin{tabular}{|c|c|c|c|c|c|}
\hline Name & Photoelectric heating & Photoionization & Supernovae & $l_{\text {gas }} / l_{\text {star }}$ & Notes \\
\hline$P E-P I-S N$ & Yes & Yes & Yes & 2 & \\
\hline PE-noPI-SN & Yes & No & Yes & 2 & \\
\hline$n o P E-P I-S N$ & No & Yes & Yes & 2 & \\
\hline noPE-noPI-SN & No & No & Yes & 2 & \\
\hline PE-noPI-noSN & Yes & No & No & 2 & \\
\hline$c P E-P I-S N$ & Yes & Yes & Yes & 2 & $G_{0}=0.1($ constant $)$ \\
\hline$P E-P I-S N-3 M y r$ & Yes & Yes & Yes & 2 & $t_{\text {life }}=3 \mathrm{Myr}($ constant $)$ \\
\hline nоPE-noPI-noSN & No & No & No & 2 & \\
\hline$P E-P I-S N-c m p$ & Yes & Yes & Yes & 1 & \\
\hline$n o P E-P I-S N-c m p$ & No & Yes & Yes & 1 & \\
\hline$P E-n o P I-n o S N-c m p$ & Yes & No & No & 1 & \\
\hline$n o P E-n o P I-S N-c m p$ & No & No & Yes & 1 & \\
\hline noPE-noPI-noSN-cmp & No & No & No & 1 & \\
\hline
\end{tabular}
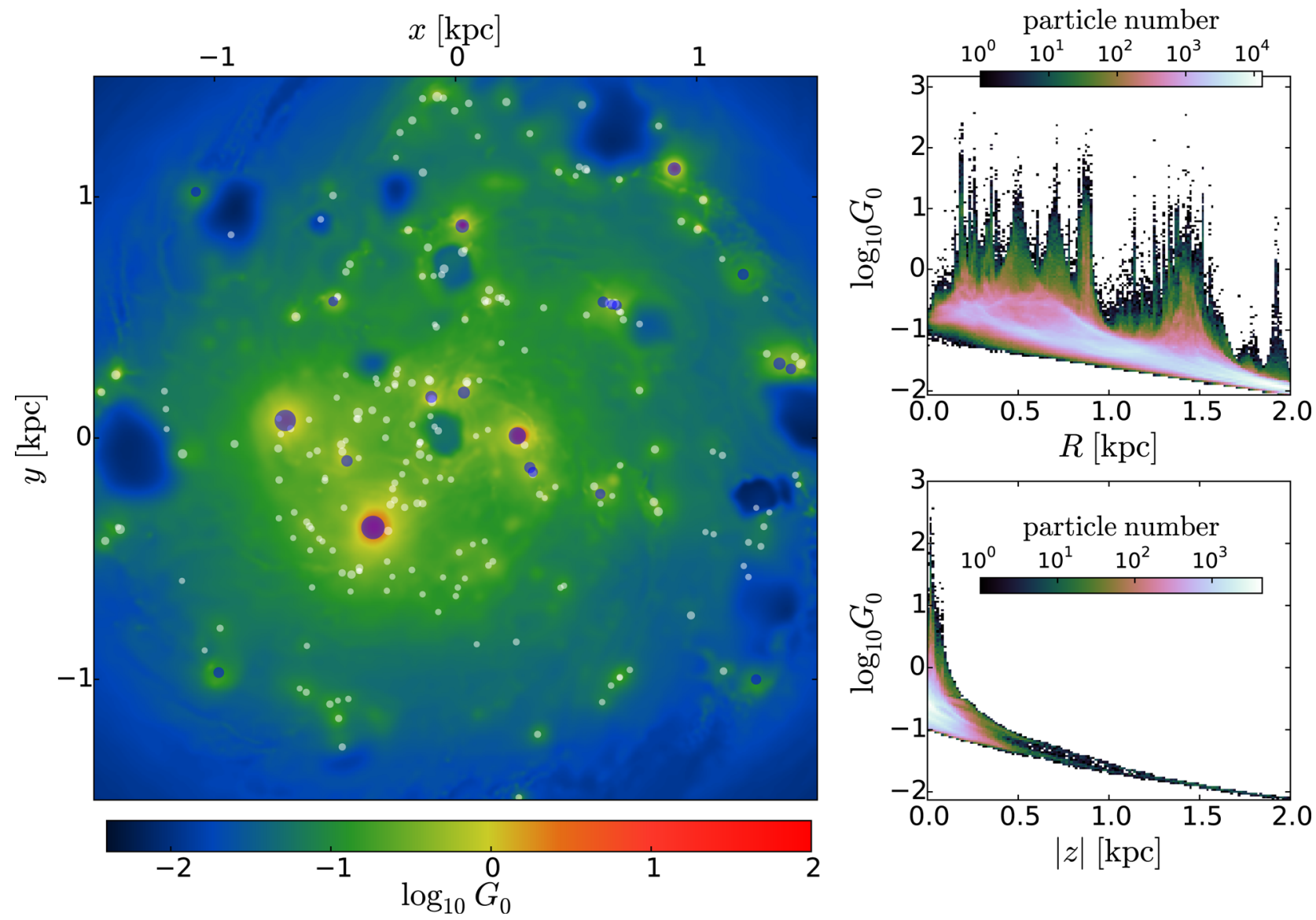

Figure 2. Left-hand panel: a face-on map of the (mass-weighted) ISRF at $z=0$ for the fiducial run PE-PI-SN at simulation time $t=600 \mathrm{Myr}$. The star particles with $m_{*}>8$ and $4 \mathrm{M}_{\odot}<m_{*} \leq 8 \mathrm{M}_{\odot}$ are overplotted in blue and white circles, respectively. Their sizes are linearly proportional to their masses. Note that the holes that associate with the feedback-driven bubbles in the map are mostly visualization artefacts as there are very few particles within the holes. Top right panel: the ISRF as a function of $R$ for gas within $|z|<0.5 \mathrm{kpc}$. Bottom right panel: the ISRF as a function of $|z|$ for gas within $R<0.5 \mathrm{kpc}$.

and because they are short-lived. Note that since the map is massweighted (hence mass-biased) the 'holes' that associate with the feedback-driven bubbles in the map are mostly visualization artefacts. To be more quantitative, the top right panel of Fig. 2 shows the ISRF as a function of $R$ for gas within $|z|<0.5 \mathrm{kpc}$, while the bottom right panel shows the ISRF as a function of $|z|$ for gas within
$R<0.5 \mathrm{kpc}$. Here the ISRF is shown directly on the particle level without mass weighting, and therefore it shows no artificial deficit within the holes. The majority of the gas is subject to the smooth ISRF, which declines with $R$ from $G_{0} \sim 0.2$ in the central region to $G_{0} \sim 0.01$ at $R=2 \mathrm{kpc}$. Along the $z$-axis the ISRF declines very rapidly as most star formation occurs within the mid-plane. Only a 

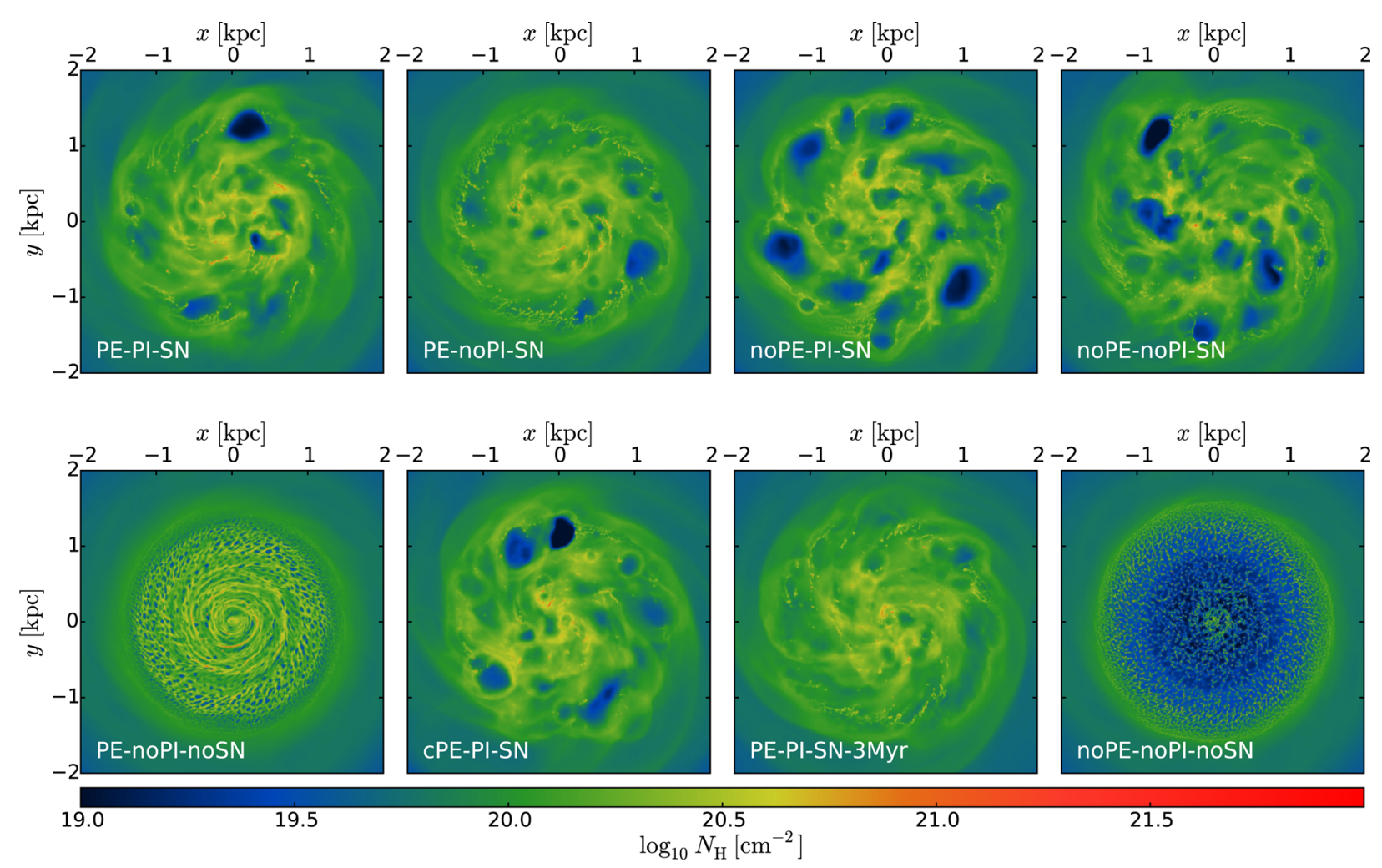

Figure 3. Face-on maps of the hydrogen nuclei column density of all runs for the fiducial disc at $t=500 \mathrm{Myr}$. In runs without SNe (PE-noPI-noSN and noPE-noPI-noSN), the morphology of the disc become very different from those including SNe. The feedback-driven bubbles show different sizes in different runs. In particular, there are almost no bubbles in PE-PI-SN-3Myr. As will be discussed in Section 3.6, this is because very few SNe go off in low-density environments.

small number of gas particles close to the UV-emitting stars have locally enhanced ISRF which can be orders of magnitude higher than the background field.

\subsection{Gas morphology}

Fig. 3 shows the face-on maps of the hydrogen nuclei column density of all runs for the fiducial disc at $t=500 \mathrm{Myr}$. All runs except for those without SNe (PE-noPI-noSN and noPE-noPI-noSN) show similar morphologies: a structured ISM with 'bubbles' driven by vigorous stellar feedback. $P E-n o P I-n o S N$ appears strikingly different from all other runs with $\mathrm{SNe}$ and it is qualitatively more similar to noPE-noPI-noSN, where the ISM is clumpier and less dynamic, and it shows little sign of disturbance from stellar feedback. This implies that PE heating is not energetic enough to trigger blast waves and inject turbulence. As shown in Fig. 1, even in the extreme conditions with very strong ISRF, the dense gas will not be heated above a few hundred of Kelvin as $T$ scales sub-linearly with $G_{0}$. This is very different from photoionization which can easily heat up the gas to about $10^{4} \mathrm{~K}$ and creates a large pressure imbalance which will subsequently drive an expansion wave. PE heating is locally a mild feedback process and thus the ISM appears more static in PE-noPI-noSN. Compared to the no-feedback run, the PE-only run shows less fragmentation as the gas is generally warmer in the presence of PE heating.

Among the other six runs with $\mathrm{SNe}$, the feedback-driven bubbles are notably smaller in both PE-noPI-SN and PE-PI-SN-3Myr. As will be discussed in Section 3.6, this is because in these two runs very few $\mathrm{SNe}$ go off at low densities. The $\mathrm{SNe}$ therefore become inefficient due to radiative energy loss and therefore very few
$\mathrm{SN}$-driven bubbles can form, even if the SFR is very similar to that in all of the other runs (see Fig. 6).

Fig. 4 shows the edge-on maps of the hydrogen nuclei number density of all runs for the fiducial disc at $t=500 \mathrm{Myr}$. The PE-only run (PE-noPI-noSN) has a significantly thinner disc compared to other $\mathrm{SN}$ runs, where the disc is puffed up by the turbulent motions triggered by SNe. A quantitative comparison of the (time-averaged) scaleheight of the gas disc will be shown in Fig. 5. SNe create bubbles that can reach high latitude and push the gas out of the disc. Again, the bubbles in both PE-noPI-SN and PE-PI-SN-3Myr are smaller compared to the other runs, as a result of having most $\mathrm{SNe}$ occurring at high densities.

\subsubsection{Scaleheight of the gaseous disc}

Fig. 5 shows the scaleheight of the gas disc as a function of $R$ in eight different runs, averaged over a period from $t=400 \mathrm{Myr}$ to $t=900 \mathrm{Myr}$ with a time interval of $10 \mathrm{Myr}$. The left-hand panel shows the runs for the fiducial disc with a scalelength of the gas disc $l_{\text {gas }}=1.46 \mathrm{kpc}$ and the right-hand panel for the compact disc with $l_{\text {gas }}=0.73 \mathrm{kpc}$. The scaleheight is defined as the latitude $z_{\mathrm{s}}$ where 75 percent of the gas is enclosed between $z= \pm z_{\mathrm{s}}$ at the given radial bin. A significant flaring can be seen in all cases. In the left-hand panel, the noPE-noPI-SN and noPE-PI-SN runs have the largest scaleheight at all radii. This implies a correlation between gas scaleheight and the $\mathrm{SN}$ rates (due to their slightly higher SFR that will be shown in Fig. 6) as the SNe enhance both the thermal (by shock heating) and kinetic (by turbulence) pressure support. Photoionization seems to play a minor role in affecting the gas scaleheight. The PE-noPI-noSN run has the lowest scale height 

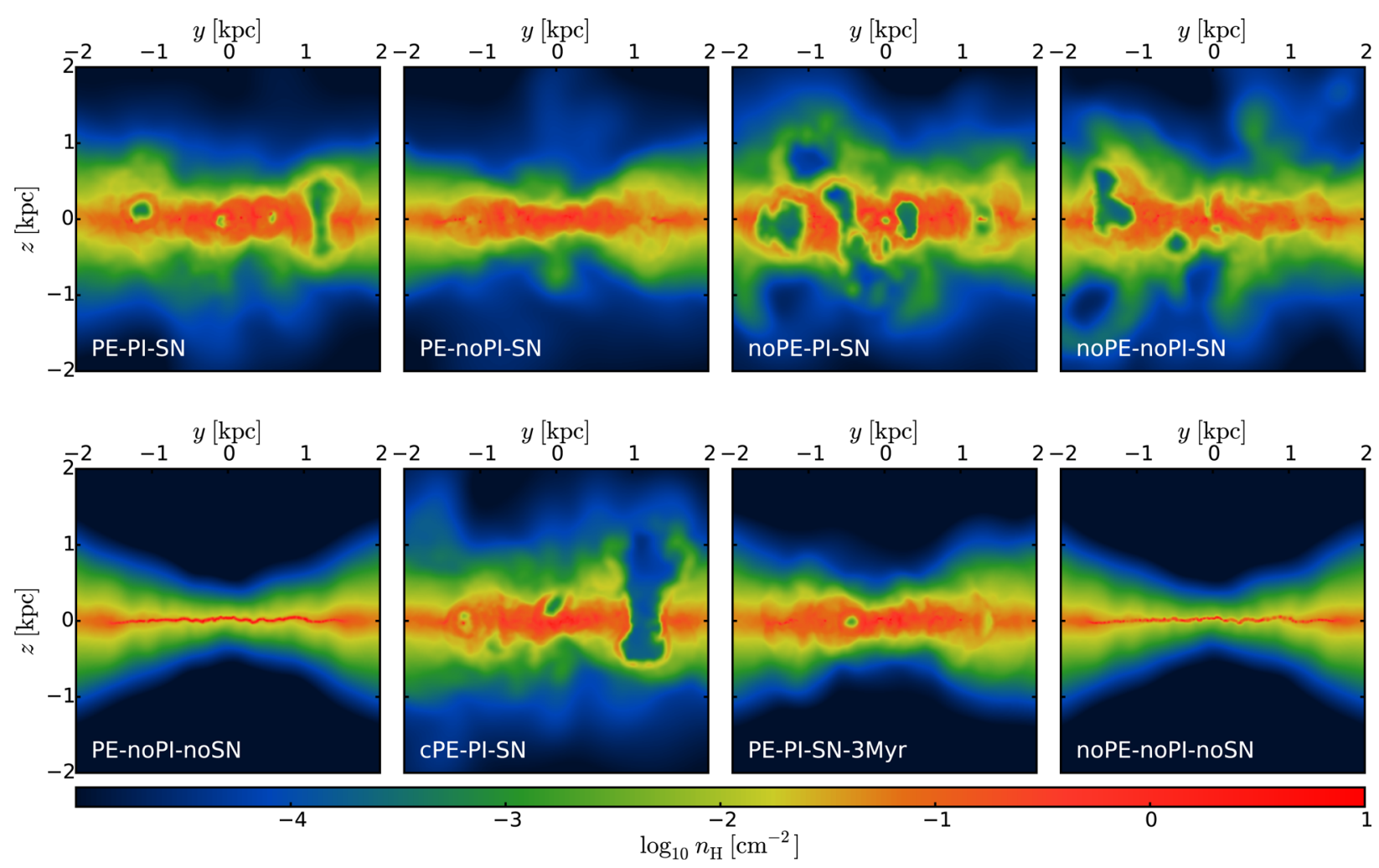

Figure 4. Edge-on maps of the hydrogen nuclei number density of all runs for the fiducial disc at $t=500 \mathrm{Myr}$. In runs without SNe (PE-noPI-noSN and noPE-noPI-noSN), we find a significantly thinner disc compared to the other runs, as in the latter the disc is puffed up by the turbulent motions triggered by $\mathrm{SNe}$. A quantitative comparison of the (time-averaged) scaleheight of the gas disc will be shown in Fig. 5. The feedback-driven bubbles can reach high latitude and push the gas out of the disc.
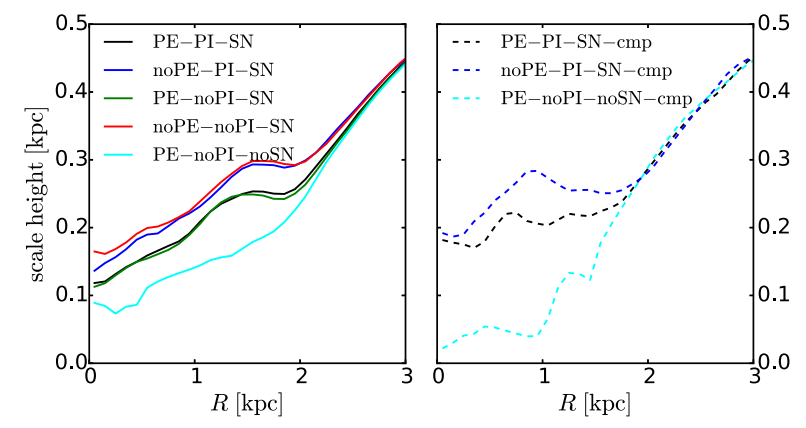

Figure 5. The scaleheight of the gaseous disc as a function of $R$ in eight different runs, averaged over a period from $t=400 \mathrm{Myr}$ to $t=900 \mathrm{Myr}$ with a time interval of $10 \mathrm{Myr}$. The left-hand panel shows the runs for the fiducial disc with a scalelength of the gas disc $l_{\text {gas }}=1.46 \mathrm{kpc}$ and the right-hand panel for the compact disc with $l_{\text {gas }}=0.73 \mathrm{kpc}$. The scaleheight increases with the SN rate, which scales with the SFR (if SNe are included) shown in Fig. 6. The lowest scaleheights are found in the runs without SNe.

because its SN rate is zero even though it has an SFR comparable to the other runs. PE heating itself does not trigger significant turbulence. In the right-hand panel, a similar trend can be seen that the scaleheight is positively correlated with the $\mathrm{SN}$ rate. In addition, turning off $\mathrm{SNe}$ in the compact disc has a much stronger effect than in the fiducial disc, as the vertical gravity is stronger in the former case. This is connected to the fact that in the absence of SN feedback, star formation can only be suppressed in the fiducial disc and not in the compact disc (see Fig. 6).

\subsection{Global properties}

\subsubsection{Total SFR}

Fig. 6 shows the total SFR of the entire galaxy as a function of time. For the fiducial disc (solid lines, upper panel), the total SFR is not very sensitive to any of the feedback combinations that we explore (their SFRs differ within a factor of two). A weak trend can still be seen: the more feedback processes we include, the lower is the SFR. noPE-noPI-SN has the highest SFR overall, which reaches a peak of $\sim 10^{-3} \mathrm{M}_{\odot} \mathrm{yr}^{-1}$ in the initial phase $(t<0.2 \mathrm{Gyr})$, but then drops by a factor of two afterwards and becomes only slightly higher than the other runs. This suggests that $\mathrm{SNe}$ alone are able to self-regulate the ISM. Switching on either photoionization (noPE$P I-S N)$ or PE heating $(P E-n o P I-S N)$ further decreases the SFR, and the SFR becomes the lowest in the 'full-feedback' run $(P E-P I-S N)$. Stellar lifetime and the distribution of $G_{0}$ appear to have negligible effect on the SFR (comparing PE-PI-SN-3Myr, $c P E-P I-S N$ and $P E$ $P I-S N)$. The SFR in PE-noPI-noSN is similar to $P E-P I-S N$ at first but it shows a gradual increase over time and at the end of the simulation $(t=1 \mathrm{Gyr})$ it is almost as high as the SFR in noPE-noPI-SN. ${ }^{9}$

The situation becomes very different in the compact disc (lower panel, dashed lines). PE-noPI-noSN-cmp shows more than an order of magnitude higher SFR than the other two runs, suggesting that $\mathrm{PE}$ heating is no longer able to suppress cooling and gravitational

\footnotetext{
${ }^{9}$ In fact, as we will show in Appendix B2, the SFR in the PE-only model will continue to rise up to $10^{-3} \mathrm{M}_{\odot} \mathrm{yr}^{-1}$ at $t=2 \mathrm{Gyr}$ and it shows no sign of saturation. Therefore, in this model the SFR depends on the 'age' of the ISM, i.e. the time period for which the galaxy has been forming stars.
} 

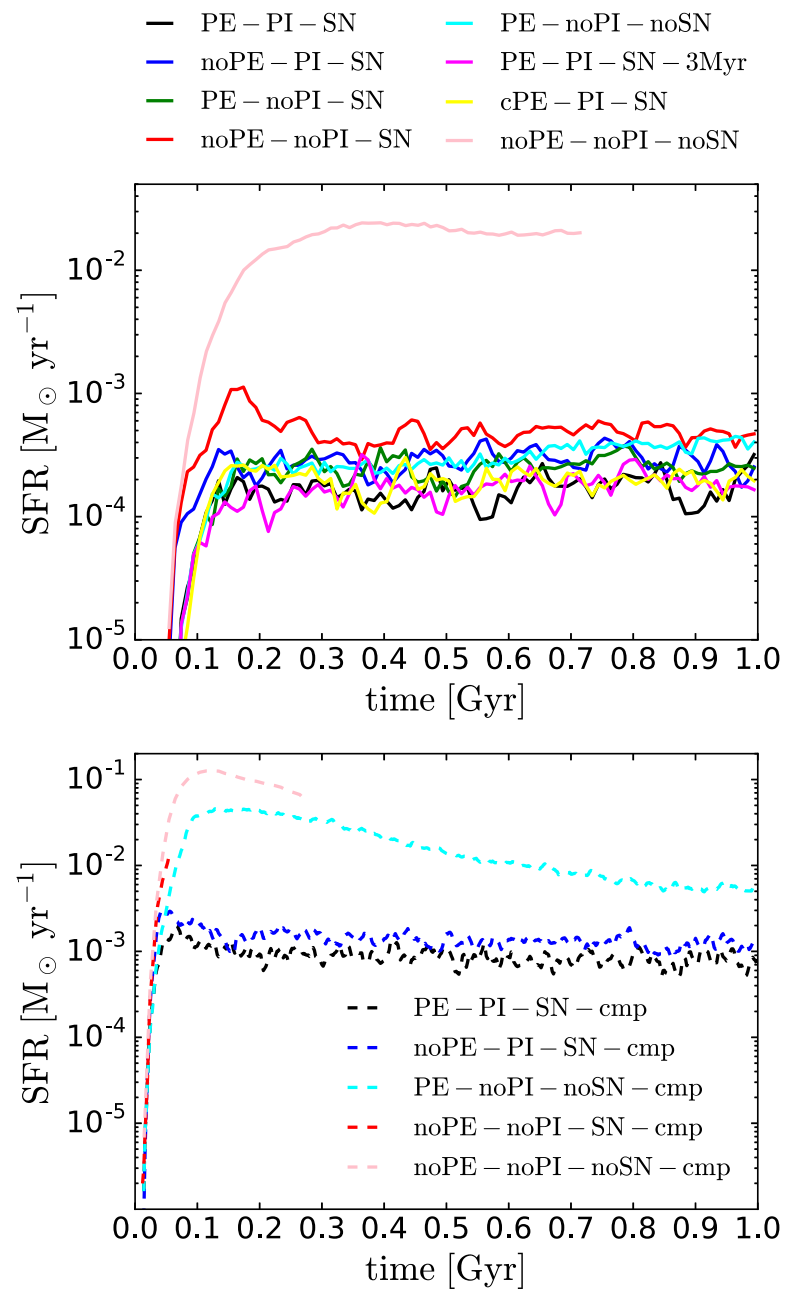

Figure 6. Time evolution of the total SFR in the fiducial (upper panel) and compact (lower panel) disc. All combinations of feedback are able to suppress star formation in the fiducial disc, including the PE-only and SN-only runs. In the compact disc, star formation can only be suppressed when both photoionization and $\mathrm{SNe}$ are included.

collapse at high gas surface densities. SNe alone (noPE-noPI-SNcmp) also cannot suppress star formation in the compact disc. Moreover, the large amount of unresolved dense gas in this run will later be heated to very high temperatures by $\mathrm{SNe}$, which leads to timesteps too small for us to continue the simulation after $t>70 \mathrm{Myr}$. As this is too short for us to draw any robust conclusion, we will not discuss noPE-noPI-SN-cmp further in this paper. Including photoionization helps suppress star formation, as $P E-P I$ $S N$-cmp and noPE-PI-SN-cmp both show much lower SFRs. This highlights the importance of feedback from massive stars prior to their SN events for regulating star formation in the higher gas surface density regime [see also Gatto et al. (2017) who investigated the importance of stellar winds in a Milky Way setup]. The fact that $P E-P I-S N$-cmp and noPE-PI-SN-cmp both have very similar SFR is consistent with our previous study in Paper I where we found that the SFR in dwarf galaxies is insensitive to the adopted value of $G_{0}$ (constant field). The two full-feedback runs with different initial conditions (PE-PI-SN and PE-PI-SN-cmp) have their SFRs differing by almost an order of magnitude due to the difference in their gas surface density: SFR scales superlinearly with the gas surface density in this regime (Bigiel et al. 2010; Schruba et al. 2011). In the absence of feedback of any kind (noPE-noPI-noSN and noPE-

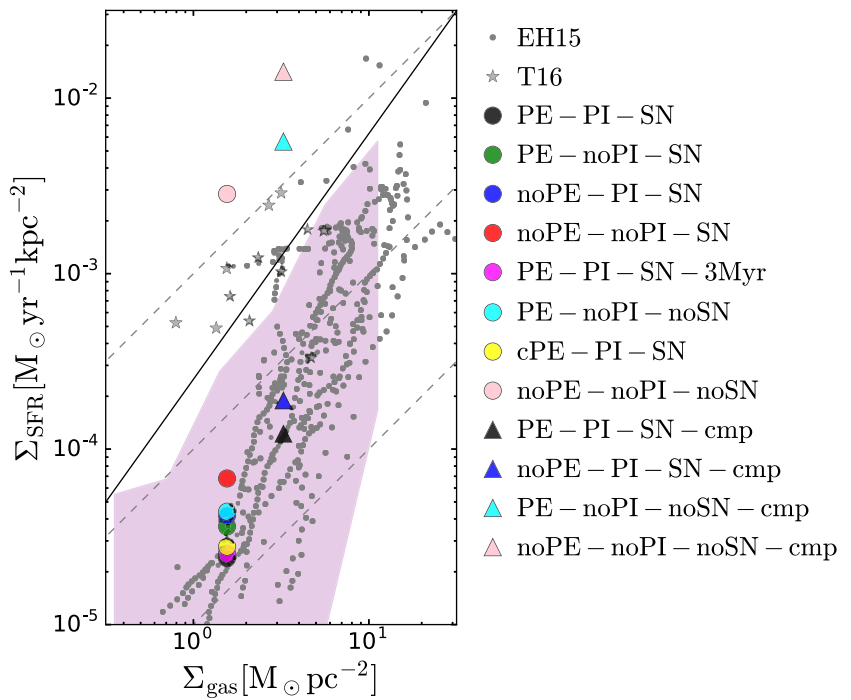

Figure 7. Comparison between the simulated galaxies and the observed dwarf galaxies on the Kennicutt-Schmidt plane (Kennicutt 1998): the star formation surface density $\left(\Sigma_{\mathrm{SFR}}\right)$ versus the gas surface density $\left(\Sigma_{\text {gas }}\right)$. The grey dots are observed data from Elmegreen \& Hunter (2015). The grey stars represent observed data from Teich et al. (2016). The purple filled area encloses the 5th and 95th percentile of the observed data from Roychowdhury et al. (2015). The solid line shows the conventional Kennicutt-Schmidt relation (Kennicutt 1998) while the dashed lines represent the gas depletion times of 1,10 and $100 \mathrm{Gyr}$ from top to bottom. All of our models except for PE-noPI-noSN-cmp and the two runs without any feedback show a reasonable amount of star formation for galaxies in this regime.

noPI-noSN-cmp), both discs collapse globally and form stars much too rapidly compared to the observed dwarf galaxies.

Fig. 7 shows the comparison between the simulated galaxies and the observed dwarf galaxies on the Kennicutt-Schmidt plane (Kennicutt 1998): the star formation surface density $\left(\Sigma_{\mathrm{SFR}}\right)$ versus the gas surface density $\left(\Sigma_{\text {gas }}\right)$. The grey dots are observed data from Elmegreen \& Hunter (2015). The grey stars represent observed data from Teich et al. (2016). The purple filled area encloses the 5th and 95th percentile of the observed data from Roychowdhury et al. (2015). The total $\Sigma_{\mathrm{SFR}}$ and $\Sigma_{\text {gas }}$ of the simulated galaxies are calculated by time-averaging over a time period from $t=200 \mathrm{Myr}$ to $t=1000 \mathrm{Myr}$ with a time interval of $10 \mathrm{Myr}$ and assuming the star-forming area is the circular area within $R<1.5 \mathrm{kpc}$. The solid line shows the conventional Kennicutt-Schmidt relation (Kennicutt 1998) while the dashed lines represent the gas depletion times of 1, 10 and $100 \mathrm{Gyr}$ from top to bottom. All of our models except for PE-noPI-noSN-cmp and the two runs without any feedback show a reasonable amount of star formation for galaxies in this regime. ${ }^{10}$

\subsection{2 $\mathrm{H}_{2}$ fraction}

The top panel of Fig. 8 shows the time evolution of the total $\mathrm{H}_{2}$ mass fraction (ratio of the mass in $\mathrm{H}_{2}$ to the total hydrogen mass) in the

${ }^{10}$ The data from Elmegreen \& Hunter (2015) and Roychowdhury et al. (2015) we compare with are in fact both spatially resolved quantities. As our purpose here is to verify whether the simulated galaxies have a long gas depletion time as seen in observations, we only present the disc-integrated quantities of the simulated galaxies rather than the pixelwise quantities as we did in Paper I, which should be sufficient for our purpose. 

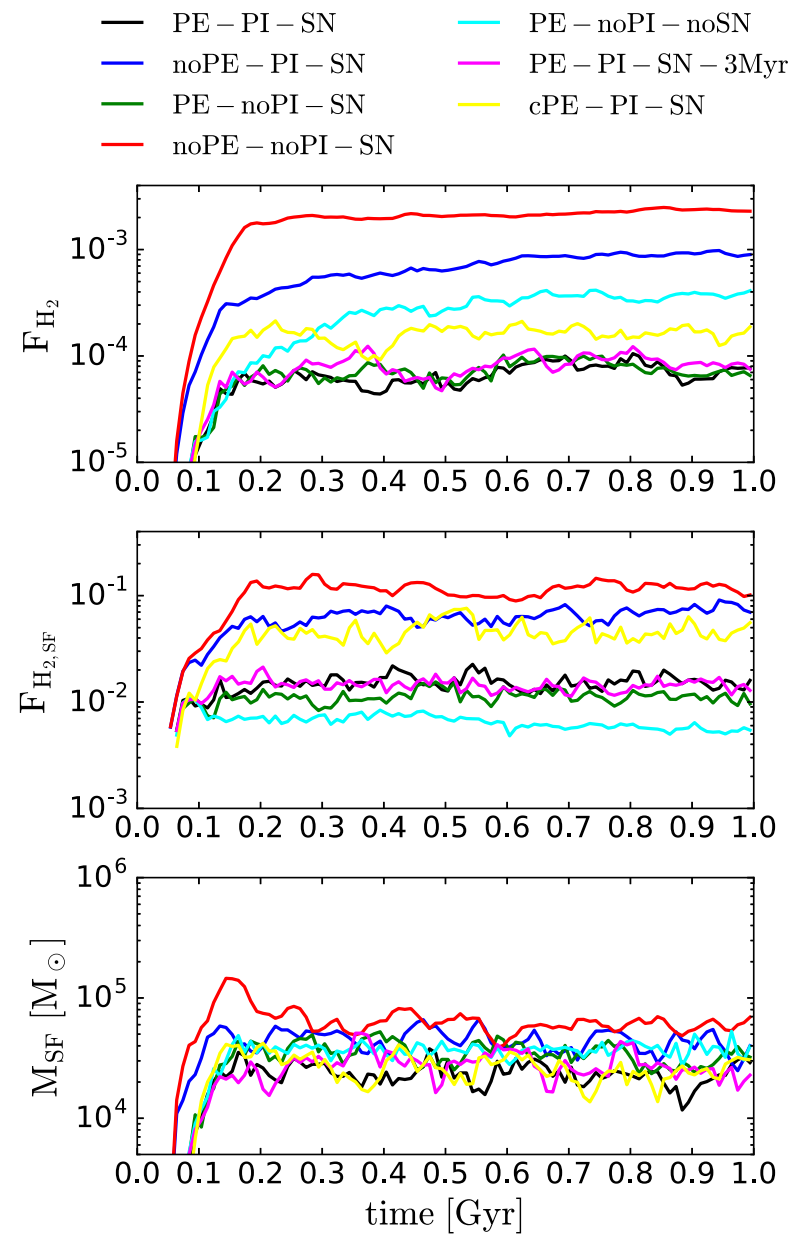

Figure 8. Top and middle panels: time evolution of the $\mathrm{H}_{2}$ mass fraction of the total gas (top) and the star-forming gas (middle) in the ISM region $(R<1.5 \mathrm{kpc}$ and $|z|<1 \mathrm{kpc})$. Bottom panel: time evolution of the total mass of the star-forming gas. The $\mathrm{H}_{2}$ fraction increases drastically when the ISRF is turned off, suggesting that photodissociation is the main destruction mechanism rather than collisional dissociation triggered by SNe. Without $\mathrm{SNe}$, however, most of the gas collapses into dense clumps which also leads to higher $\mathrm{H}_{2}$ fraction. Star formation is mainly controlled by the amount of gas that can condense into the cold and dense phase, which is set by the competition between gravity and stellar feedback.

ISM region. The three runs with both variable PE heating and $\mathrm{SNe}$ all show comparable low $\mathrm{H}_{2}$ fractions, and the $c P E-P I-S N$ run has a slightly higher $\mathrm{H}_{2}$ fraction due to its lack of strong radiation fields in the vicinity of young stars which can efficiently destroy $\mathrm{H}_{2}$. Once PE heating is switched off, the $\mathrm{H}_{2}$ fraction increases drastically by more than one order of magnitude. However, this is an artificial consequence of our implementation: we switch off PE heating by setting $G_{0}=0$, but this also disables $\mathrm{H}_{2}$ photodissociation, and it is the lack of this process that leads to the large change in the $\mathrm{H}_{2}$ fraction. This demonstrates that the collisional dissociation from $\mathrm{SNe}$ is a sub-dominant destruction mechanism for $\mathrm{H}_{2}$ compared to photodissociation. The noPE-PI-SN run has a lower $\mathrm{H}_{2}$ fraction than noPE-noPI-SN due to our treatment of photoionization, which destroys all $\mathrm{H}_{2}$ within the affected radius. On the other hand, the $P E-n o P I-n o S N$ run also has a high $\mathrm{H}_{2}$ fraction even with the ISRF turned on because there is much more cold and dense gas in this run (see Section 3.4). The fact that all runs shown in Fig. 8 have very different $\mathrm{H}_{2}$ fractions but almost the same SFR demonstrates that $\mathrm{H}_{2}$

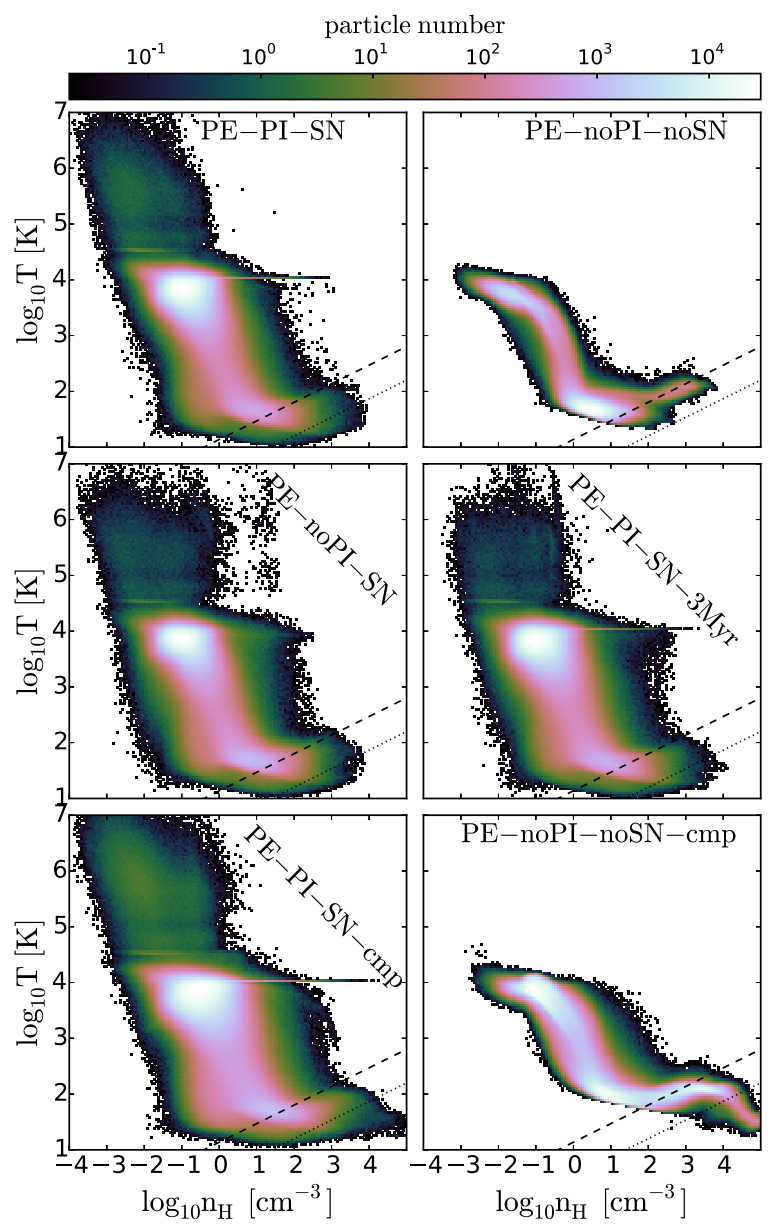

Figure 9. Phase diagrams (temperature versus number density) for the gas in the ISM region in all the runs that include self-consistent PE heating, averaged over a period from $t=400 \mathrm{Myr}$ to $t=900 \mathrm{Myr}$ with a time interval of 10 Myr. The PE-only runs show significantly different distributions from the other runs: they have much more cold gas and have no hot gas at all, and the star-forming gas is notably warmer than in the other runs. The dashed lines represent our star formation threshold where $M_{\mathrm{J}}=8 M_{\mathrm{ker}}$ while the dotted lines represent the resolution limit where $M_{\mathrm{J}}=M_{\mathrm{ker}}$.

does not trace star formation in dwarf galaxies as it does in spiral galaxies, consistent with our findings in Paper I and with prior theoretical predictions (Glover \& Clark 2012b; Krumholz 2012). Instead, star formation is mainly controlled by the amount of gas that can condense into the cold and dense phase, which is set by the competition between gravity and stellar feedback. The bottom panel of Fig. 8 shows the time evolution of the total mass of the star-forming gas $\left(M_{\mathrm{SF}}\right)$, which has a very similar behaviour to the time evolution of the SFR (see Fig. 6).

The middle panel of Fig. 8 shows the time evolution of the $\mathrm{H}_{2}$ mass fraction in the star-forming gas. In all runs including those with the ISRF switched off, the star-forming gas is dominated by the atomic hydrogen instead of $\mathrm{H}_{2}$. The ranking of different runs remains in the same order except for PE-noPI-noSN, which is related to the fact that $P E-n o P I-n o S N$ has a very different morphological structure (see Fig. 3): there are many more dense clumps compared to the other runs which leads to a higher total $\mathrm{H}_{2}$ fraction. However, these dense clumps are located very close to the UV-emitting stars (see Fig. 9) and therefore are illuminated by a much stronger ISRF which leads to the lowest $\mathrm{H}_{2}$ fraction in star-forming gas in all runs. 


\subsection{Local properties}

Fig. 9 shows the gas distribution on the phase diagram (number density versus temperature) in the ISM region in all six runs that include self-consistent PE heating, averaged over a period from $t=$ $400 \mathrm{Myr}$ to $t=900 \mathrm{Myr}$ with a time interval of $10 \mathrm{Myr}$. The dotted lines show the contours where $M_{\mathrm{J}}=M_{\mathrm{ker}}=400 \mathrm{M}_{\odot}$, which can be viewed as our resolution limit. The dashed lines show the star formation threshold, $M_{\mathrm{J}}=8 M_{\mathrm{ker}}$. The two runs with only PE heating (PE-noPI-noSN and PE-noPI-noSN-cmp) show very different distributions from the other runs: there is much more cold gas when PE heating is the only feedback mechanism. In the fiducial disc (PE-noPI-noSN), PE heating alone is able to heat up the dense gas to $T \gtrsim 100 \mathrm{~K}$, which appears to be enough to stop further gravitational collapse and therefore maintain a low SFR. ${ }^{11}$ However, for the compact disc (PE-noPI-noSN-cmp), a significant amount of gas collapses to very high densities $\left(n \gtrsim 10^{3}-10^{4} \mathrm{~cm}^{-3}\right)$ even though the gas is also heated up to $T \gtrsim 100 \mathrm{~K}$ by PE heating, and thus the SFR is boosted dramatically (see Fig. 6).

The other four runs with SNe in Fig. 9 have a more scattered distribution, mainly due to enhanced turbulent motions driven by SNe. These runs also have hot gas $\left(T>3 \times 10^{4} \mathrm{~K}\right)$ which can only be generated by $\mathrm{SNe}$. The dense gas in these runs has a slightly cooler gas temperature on average (below $100 \mathrm{~K}$ ). The narrow lines at $T=10^{4} \mathrm{~K}$ are due to photoionization. The majority of gas is warm ( $\left.T \sim 10^{4} \mathrm{~K}\right)$. Notably, it is much warmer than its thermal equilibrium temperature (shown in Fig. 1), as the gas is constantly stirred and shocked by both $\mathrm{SNe}$ and photoionization and has insufficient time to cool. As shown in Paper I, this occurs when the gas cooling time is longer than the turbulent dynamical time, which is more likely to be the case in low-metallicity systems. In PE-PI-SN-cmp, the gas retained at $10^{4} \mathrm{~K}$ extends to slightly higher densities compared to the other three runs because of the higher SFR and therefore higher $\mathrm{SN}$ rate.

Fig. 10 shows the ISM distribution on a $n-G_{0}$ plane (number density versus ISRF) for the same set of runs as in Fig. 9, averaged over the same time period from $t=400 \mathrm{Myr}$ to $t=900 \mathrm{Myr}$ with a time interval of $10 \mathrm{Myr}$. In the two PE-only runs (PE-noPI-noSN and $P E-n o P I-n o S N-c m p)$, the dense gas is illuminated by a very strong ISRF with orders of magnitude higher $G_{0}$ compared to that in the other four runs. This is responsible for the much higher temperatures found in the star-forming gas in these runs (see Fig. 9). The lack of $\mathrm{SNe}$ (and/or photoionization) results in a less turbulent ISM where most of the gas will simply be attracted towards the star-forming clumps due to gravity. Therefore, the gas experiences a much higher $G_{0}$ due to its proximity to the UV-emitting stars. On the other hand, once $\mathrm{SNe}$ are included, they trigger blastwaves and turbulence which disperse the nearby clouds. As such, the dense gas on average is further away from the sources, and so the typical $G_{0}$ found in the dense gas drops significantly. This means that PE heating in these runs is actually much weaker than it is in the PEonly runs, where the omission of other feedback mechanisms ( $\mathrm{SNe}$ and photoionization) turns out to significantly boost the effect of PE heating. This effect is mainly caused by a change in the gas distribution and not by the energy budget of the ISRF (as the total SFR is almost unchanged).

Fig. 11 shows the phase diagrams of the ISM in the runs with variable $G_{0}$ (upper left), constant $G_{0}$ (upper right) and $G_{0}=0$

\footnotetext{
${ }^{11}$ The suppression of the SFR of the PE-only run has a slight dependence (around a factor of two) on our choice of the gravitational softening length (see Appendix B2).
}

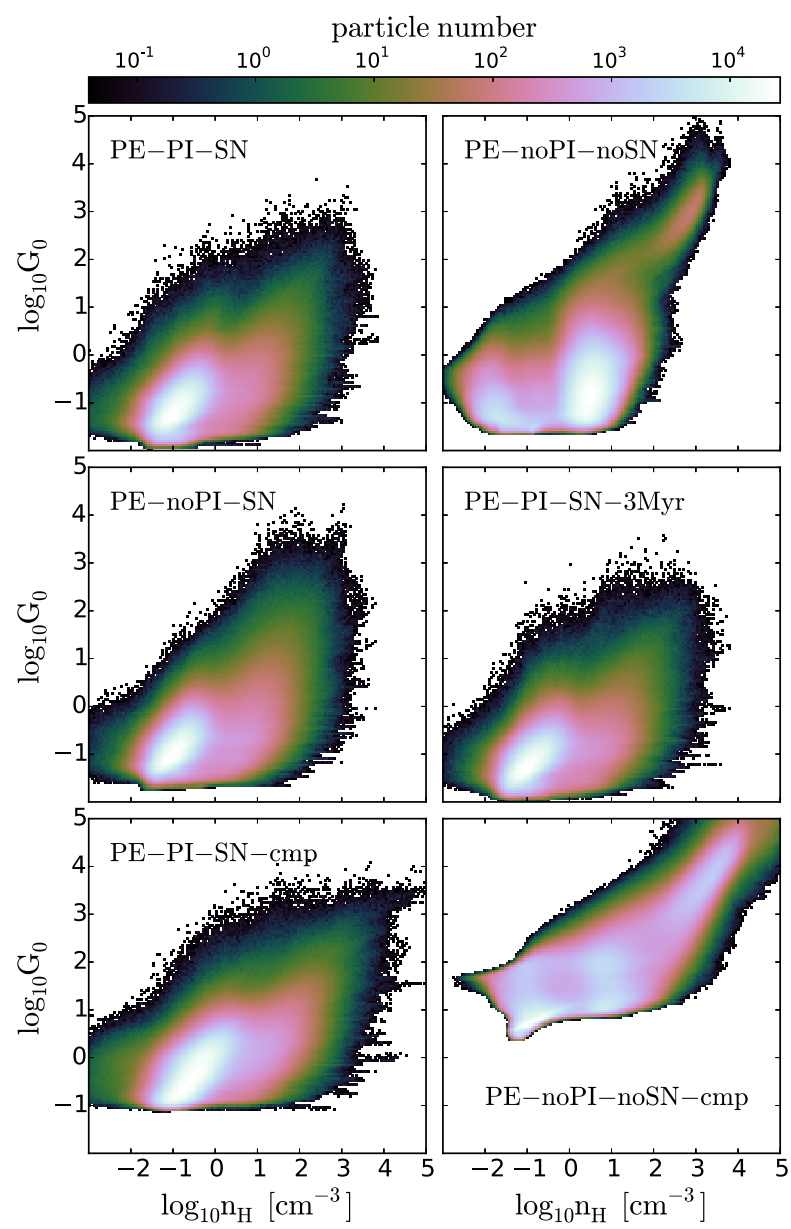

Figure 10. The strength of the ISRF $\left(G_{0}\right)$ versus the hydrogen number density $\left(n_{\mathrm{H}}\right)$ for gas in the ISM region, averaged over a period from $t=$ $400 \mathrm{Myr}$ to $t=900 \mathrm{Myr}$ with a time interval of $10 \mathrm{Myr}$. Dense gas in the runs with $\mathrm{PE}$ heating alone is subject to much higher $G_{0}$ as the dense clumps are much closer to the radiation sources. With $\mathrm{SNe}$ the gas is dispersed and so $G_{0}$ is much lower.

(lower panels), averaged over a period from $t=400 \mathrm{Myr}$ to $t=$ $900 \mathrm{Myr}$ with a time interval of $10 \mathrm{Myr}$. The gas distribution is similar in all of these runs, with the majority of the gas being warm $\left(T \sim 10^{4} \mathrm{~K}\right)$ and diffuse $\left(n \sim 0.1 \mathrm{~cm}^{-3}\right)$. The main difference is that without PE heating (noPE-PI-SN and noPE-noPI-SN) the cold gas can cool down to slightly lower temperatures (by $\sim 0.5 \mathrm{dex}$ ). This makes the system more gravitationally unstable and results in more star-forming gas, which manifests as a 'tail' in the phase diagram that further extends into the dense unresolved regions (to the right of the dotted line). Switching on the (variable) PE heating $(P E-P I-S N)$ makes most of the star-forming gas stay at the resolved region. This difference in the star-forming gas leads to the slightly higher total SFR in Fig. 6 for the no PE heating runs. The constant- $G_{0}$ run ( $\left.c P E-P I-S N\right)$ shows a behaviour intermediate between the variable- $G_{0}$ run and the no- $G_{0}$ run: it has a slightly warmer cold gas due to the PE heating from the background radiation field, but it also has a high-density tail as there is no enhanced PE heating in the densest gas. In all cases, $\mathrm{SNe}$ are able to keep the majority of gas warm $\left(\sim 10^{4} \mathrm{~K}\right)$ even in the SN-only run (noPE-noPI-SN). 


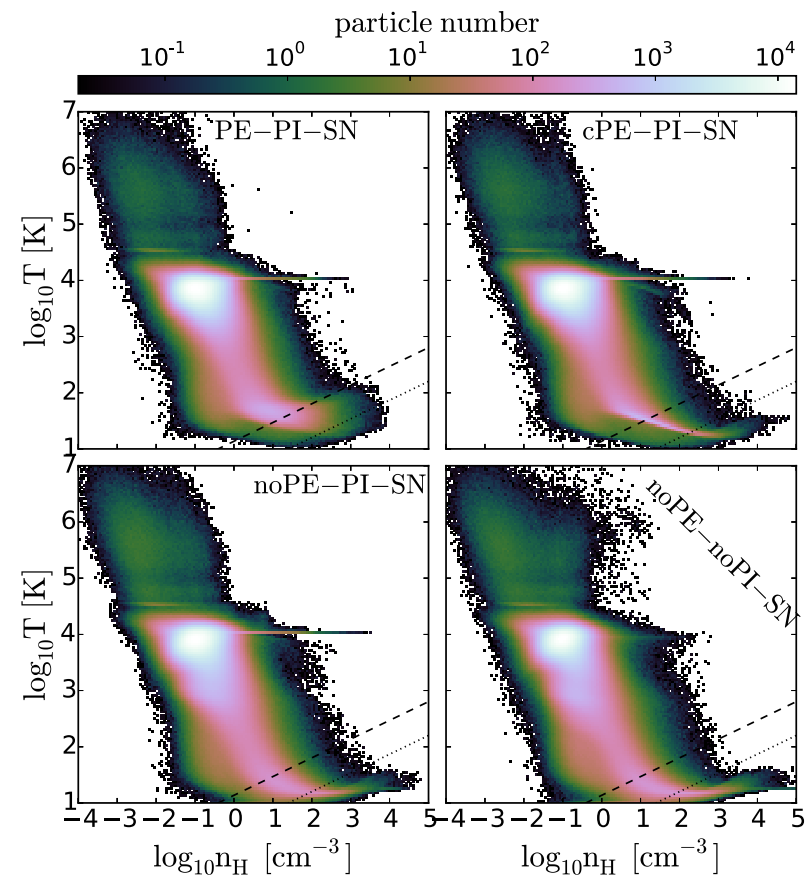

Figure 11. Phase diagrams of the ISM in the runs with variable $G_{0}$ (upper left), constant $G_{0}$ (upper right) and $G_{0}=0$ (lower panels), averaged over a period from $t=400 \mathrm{Myr}$ to $t=900 \mathrm{Myr}$ with a time interval of $10 \mathrm{Myr}$. Without variable PE heating, the cold gas is slightly colder, but the overall distribution does not change much. $\mathrm{SNe}$ are able to keep the majority of gas warm $\left(\sim 10^{4} \mathrm{~K}\right)$ even without PE heating, which is consistent with our findings in Paper I.

\subsection{FIR lines and IR continuum emission}

We estimate the total luminosity from the far-infrared (FIR) fine structure emission lines [C II] $157 \mu \mathrm{m},\left[\mathrm{O}_{\mathrm{I}}\right] 63 \mu \mathrm{m}$ and [O I] $145 \mu \mathrm{m}$ for the simulated galaxies via post-processing, denoted as $L_{\text {[C II]157 }}, L_{\text {[O I]63 }}, L_{\text {[O I]145, }}$, respectively. The emissivity of the FIR lines for each gas particle is obtained using our non-equilibrium cooling module albeit assuming optically thin conditions, which is a fair approximation for low-metallicity gas. ${ }^{12}$ We then obtain the total luminosity by summing over the contributions of all gas particles within the circular area $R<1.5 \mathrm{kpc}$.

In addition, we estimate the continuum infrared emission from dust. The local dust temperature in our simulations is mainly dictated by dust heating due to the local ISRF and the radiative cooling of dust, the latter of which is obtained using the fitting formula given by Glover \& Clark (2012a):

$\Lambda_{\text {dust }}\left(T_{\mathrm{d}}\right)=4.68 \times 10^{-31} D^{\prime} T_{\mathrm{d}}^{6} n_{\mathrm{H}} \mathrm{erg} \mathrm{s}^{-1} \mathrm{~cm}^{-3}$,

where $D^{\prime}$ is the DGR relative to the solar value, $T_{\mathrm{d}}$ is the dust temperature and $n_{\mathrm{H}}$ is the hydrogen nuclei number density. Assuming

12 The optically thin approximation breaks down at high column densities. For example, for $\left[\mathrm{C}_{\mathrm{II}}\right] 157 \mu \mathrm{m}$ this occurs at a total hydrogen column density $N_{\mathrm{H}} \sim 10^{22} \mathrm{~cm}^{-2}$ given our $0.1 \mathrm{Z}_{\odot}$ metallicity and an assumed [C II] linewidth of $2.4 \mathrm{~km} \mathrm{~s}^{-1}$, and at a proportionately higher column density if the line-width is larger than this value. A similar column density is required to produce $\tau \sim 1$ in the [O I] $63 \mu \mathrm{m}$ line (Glover \& Smith 2016). These values are 10 times higher than the column densities found in our setups, even in our compact disc simulations. In the very dense gas where this approximation does break down we will overestimate the luminosity, but this will only affect a very small fraction of the gas in our simulations.
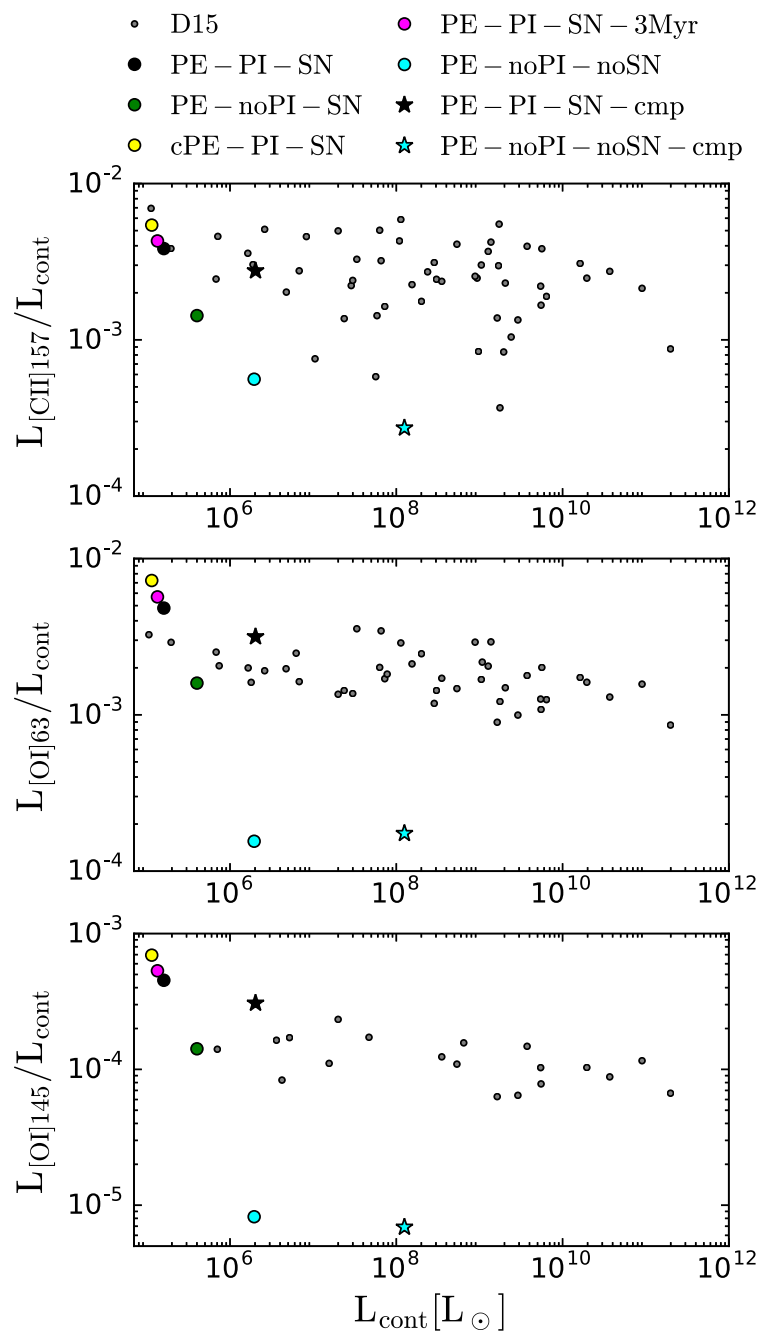

Figure 12. The total luminosity of the continuum infrared emission $L_{\text {cont }}$ versus the ratio of FIR lines to the continuum for all runs with PE heating, time-averaged over a time period from $t=200 \mathrm{Myr}$ to $t=1000 \mathrm{Myr}$. The observed data from the Dwarf Galaxy Survey from Cormier et al. (2015) are overplotted as grey dots. The PE-only runs have their FIR-to-continuum ratios much lower than the observed dwarf galaxies, especially for the oxygen lines. Including $\mathrm{SNe}$ brings the simulated galaxies into locations in the plot that agree well with observations by pushing gas further away from the massive stars.

optically thin conditions, the continuum infrared emission ( $\left.L_{\text {cont }}\right)$ is obtained by summing over the contributions of all gas particles within the circular area $R<1.5 \mathrm{kpc}^{13}$

Fig. 12 shows the relation between $L_{\text {cont }}$ and the ratio of FIR lines to the dust continuum for all runs with $\mathrm{PE}$ heating, averaged over a time period from $t=200 \mathrm{Myr}$ to $t=1000 \mathrm{Myr}$ with a time interval of 10 Myr. The observed data from the Dwarf Galaxy Survey (Cormier et al. 2015) are overplotted as grey dots. Since the noPE runs have $G_{0}=0$ everywhere and thus have no dust heating due to the ISRF, their $L_{\text {cont }}$ values are around four orders of magnitude lower than in runs with PE heating and therefore are not visible in Fig. 12. We

${ }^{13}$ Note that as equation 7 was derived assuming Ossenkopf \& Henning (1994) dust opacities, we are implicitly assuming here that in our low metallicity dwarf galaxy, the dependence of opacity on wavelength has the same form, and it is only the normalization that is different owing to the lower DGR. 
Table 2. The time-averaged (over a time period from $t=200 \mathrm{Myr}$ to $t=1000 \mathrm{Myr}$ ) total luminosities of the continuum dust emission $\left(L_{\text {cont }}\left[\mathrm{L}_{\odot}\right]\right)$ and FIR lines $\left(L_{[\mathrm{C} \mathrm{II}] 157}, L_{[\mathrm{O} \mathrm{I}] 63}\right.$ and $\left.L_{[\mathrm{O} \text { I] } 145}\right)$ as well as their fluctuations in all runs.

\begin{tabular}{lllll}
\hline Name & $L_{\text {cont }}\left(\mathrm{L}_{\odot}\right)$ & $L_{[\mathrm{CI}] 157}\left(\mathrm{~L}_{\odot}\right)$ & $L_{[\mathrm{OI}] 63}\left(\mathrm{~L}_{\odot}\right)$ & $L_{[\mathrm{OI}] 145}\left(\mathrm{~L}_{\odot}\right)$ \\
\hline PE-PI-SN & $(1.6 \pm 0.41) \times 10^{5}$ & $(610 \pm 150)$ & $(770 \pm 150)$ & $(72 \pm 17)$ \\
PE-noPI-SN & $(4.0 \pm 1.5) \times 10^{5}$ & $(570 \pm 76)$ & $(640 \pm 62)$ & $(57 \pm 5.4)$ \\
noPE-PI-SN & $(23 \pm 4.0)$ & $(630 \pm 220)$ & $(860 \pm 260)$ & $(83 \pm 28)$ \\
noPE-noPI-SN & $(22 \pm 16)$ & $(370 \pm 47)$ & $(560 \pm 77)$ & $(51 \pm 6.8)$ \\
PE-PI-SN-3Myr & $(1.3 \pm 0.41) \times 10^{5}$ & $(580 \pm 150)$ & $(760 \pm 170)$ & $(71 \pm 19)$ \\
PE-noPI-noSN & $(2.0 \pm 0.56) \times 10^{6}$ & $(1100 \pm 140)$ & $(300 \pm 47)$ & $(16 \pm 5.5)$ \\
cPE-PI-SN & $(1.1 \pm 0.03) \times 10^{5}$ & $(620 \pm 220)$ & $(830 \pm 240)$ & $(79 \pm 27)$ \\
PE-PI-SN-cmp & $(2.0 \pm 0.36) \times 10^{6}$ & $(5.6 \pm 1.2) \times 10^{3}$ & $(6.4 \pm 1.7) \times 10^{3}$ & $(540 \pm 170)$ \\
noPE-PI-SN-cmp & $(120 \pm 28)$ & $(4.8 \pm 1.0) \times 10^{3}$ & $(5.4 \pm 1.2) \times 10^{3}$ & $(860 \pm 820)$ \\
PE-noPI-noSN-cmp & $(1.3 \pm 1.2) \times 10^{8}$ & $(3.4 \pm 2.6) \times 10^{4}$ & $(2.2 \pm 2) \times 10^{4}$ & \\
\hline
\end{tabular}

report the averaged $L_{\text {cont }}$ and the FIR line luminosities as well as their fluctuations in all runs in Table 2.

For the fiducial disc, $P E-P I-S N$ and $P E-P I-S N-3 M y r$ show similar continuum and FIR line luminosities. This is unsurprising, as their gas distributions in the $n_{\mathrm{H}}-G_{0}$ plane and in the phase diagram are similar. $L_{\text {cont }}$ in PE-noPI-SN is about 2.5 higher than in PE-PI-SN as in the former case there is slightly more dense gas close to the massive stars that experiences a stronger ISRF. On the other hand, the FIR line luminosities in $P E-n o P I-S N$ do not differ much from those in $P E-P I-S N$, which implies that FIR lines mainly originate from the diffuse gas (cf. Fig. 13). In the constant- $G_{0}$ run ( $c P E-P I-$ $S N), L_{\text {cont }}$ is about 70 percent of that in $P E-P I-S N$, as the former lacks the contributions from the high- $G_{0}$ regions close to massive stars. It also has comparable FIR line luminosities to those in $P E-P I-S N$, for the same reason that the FIR lines mainly come from the diffuse gas which does not differ much in these runs.

Most strikingly, $L_{\text {cont }}$ in PE-noPI-noSN is an order of magnitude higher than in $P E-P I-S N$, as there is a significant fraction of high- $G_{0}$ gas in the former case (see Fig. 10). It also has a higher $L_{[\mathrm{C} \text { II] } 157}$ and lower $\left.L_{[\mathrm{O}}\right] 63$ and $\left.L_{[\mathrm{O}}\right] 145$ than in PE-PI-SN as a result of being dominated by cold gas rather than by warm gas. In the compact disc, the difference in $L_{\mathrm{cont}}$ between PE-PI-SN-cmp and PE-noPI-noSNcmp is even more pronounced: it is two orders of magnitude higher in the latter case which has a huge number of dense clumps with high $G_{0}$. The FIR-to-continuum ratios in the PE-only runs are much lower than the values observed in real dwarf galaxies, especially for the oxygen lines. Including SNe brings the simulated galaxies into the locations that agree well with observations by pushing gas further away from the massive stars.

\subsubsection{Cumulative IR emissions}

Fig. 13 shows the normalized cumulative IR continuum emission from dust and the $\left[\mathrm{C}_{\mathrm{II}}\right] 157 \mu \mathrm{m}$ line as a function of hydrogen number density ( $n_{\mathrm{H}}$, top row), gas temperature ( $T$, middle row) and local radiation field ( $G_{0}$, bottom row), averaged over a time period from $t=200 \mathrm{Myr}$ to $t=1000 \mathrm{Myr}$ with a time interval of $10 \mathrm{Myr}$. Only runs with $\mathrm{PE}$ heating are shown. The oxygen lines (not shown) have a similar trend as the $[\mathrm{C}$ II $] 157 \mu \mathrm{m}$ line. Runs with SNe, including the full-feedback models ( $P E$-PI-SN and PE-PI-SN-cmp), have their FIR metal lines primarily coming from the diffuse $\left(n_{\mathrm{H}}<1 \mathrm{~cm}^{-3}\right)$ and warm $\left(T>3 \times 10^{3} \mathrm{~K}\right)$ phase of the ISM. The ISM in these runs is dominated by the warm and diffuse phase both in volume and in mass, as will be shown in Fig. 16. PE-noPI-SN has around 20 per cent higher contribution from the cold phase than the fiducial run $P E-P I-S N$ because it has more cold and dense gas which

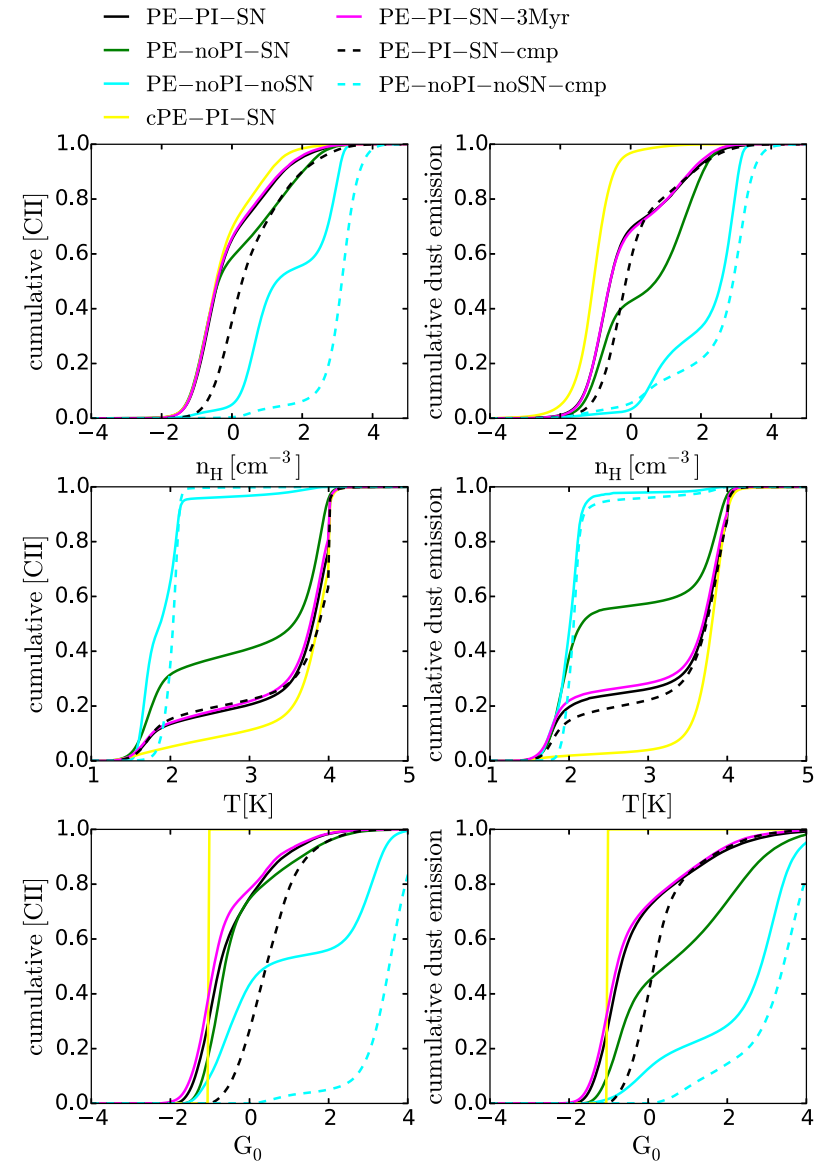

Figure 13. Normalized cumulative IR continuum emission from the $\left[\mathrm{C}_{\mathrm{II}}\right] 157 \mu \mathrm{m}$ line and from dust as a function of hydrogen number density $\left(n_{\mathrm{H}}\right.$, top row), gas temperature ( $T$, middle row) and local radiation field $\left(G_{0}\right.$, bottom row). Runs with SNe have their FIR metal lines primarily coming from the diffuse $\left(n_{\mathrm{H}}<1 \mathrm{~cm}^{-3}\right)$ and warm $\left(T>3 \times 10^{3} \mathrm{~K}\right)$ phase of the ISM. On the other hand, due to the very different gas morphology in the PE-only runs (PE-noPI-noSN and PE-noPI-noSN-cmp), most of the FIR lines and the continuum are coming from the cold and dense gas exposed to a strong ISRF in these runs.

is effectively destroyed by photoionization in the latter case. As our simple treatment of photoionization is likely to have an excessive effect on destroying dense gas, we expect that a more realistic ISM should be intermediate between PE-PI-SN and PE-noPI-SN. The FIR lines do not trace the regions where the ISRF is strong, as the majority of the emission is coming from gas with a $G_{0}$ not 

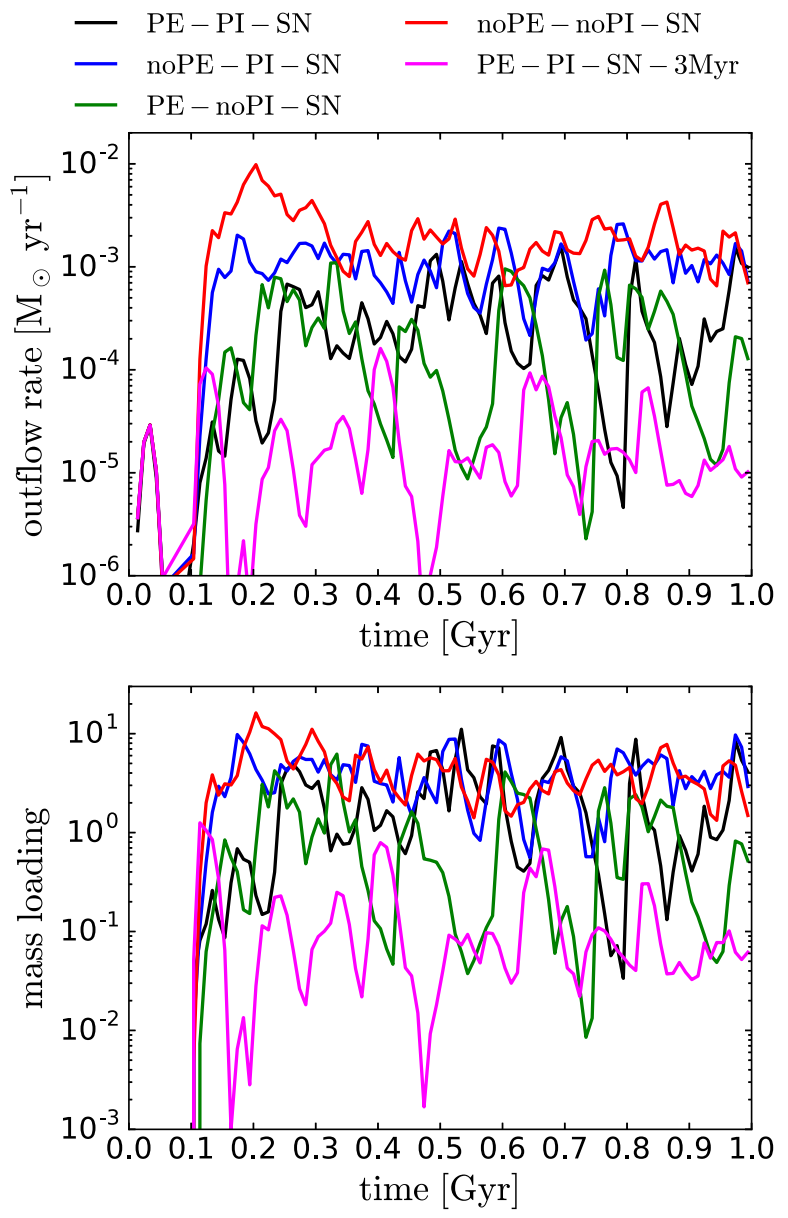

Figure 14. Time evolution of the outflow rate (upper panel) and the massloading factor (the ratio of the outflow rate to the SFR, lower panel), measured at $|z|=2 \mathrm{kpc}$. The outflow rate is much more sensitive to the adopted feedback compared to the SFR (see Fig. 6).

much higher than the background value $\left(G_{0} \sim 0.1\right.$ for the fiducial disc). The dust continuum emission originates from slightly denser and cooler gas which experiences a stronger ISRF. On the other hand, due to the very different gas morphology in the PE-only runs (PE-noPI-noSN and PE-noPI-noSN-cmp), most of the FIR lines and the continuum are coming from the cold and dense gas with a strong ISRF in these runs. We caution that our simulations do not resolve the internal structure of the dense clouds and therefore may underestimate the emission from those regions if there is, as inferred from observations such as Cormier et al. (2015) and Cigan et al. (2016), significant clumping of gas at the scales below our resolution. However, we expect that the systematic trends between different runs will be qualitatively unchanged.

\subsection{Galactic outflows}

In the upper panel of Fig. 14 we show the galactic outflow rate for the fiducial disc as a function of time. The outflow rate is defined in the same way as in Paper I, which measures the mass flux through the two planes at $z= \pm 2 \mathrm{kpc}$ along the outflowing directions. Compared to the SFR, the outflow rate fluctuates much more over time, and it is quite sensitive to the adopted feedback model. Run PE-noPI$n o S N$ is not shown because its outflow rate is essentially zero. In the lower panel of Fig. 14, we show the mass-loading factor, defined as
$\square \mathrm{PE}-\mathrm{PI}-\mathrm{SN}$
noPE-noPI-SN
$\square$ noPE-PI-SN
$\mathrm{PE}-\mathrm{PI}-\mathrm{SN}-3 \mathrm{Myr}$
$\square \mathrm{PE}-\mathrm{noPI}-\mathrm{SN}$

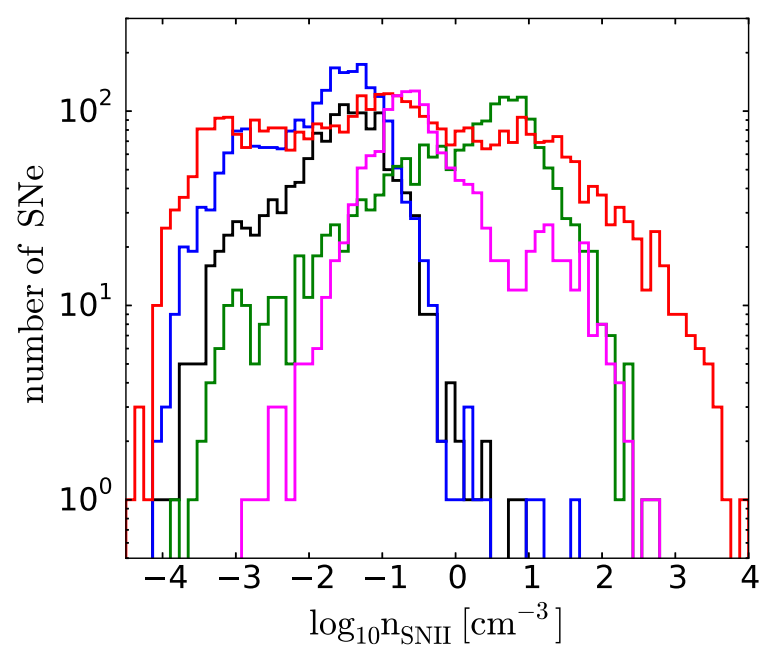

Figure 15. Histogram of the ambient hydrogen number density where the $\mathrm{SNe}$ occur $\left(n_{\mathrm{SNII}}\right)$. The more $\mathrm{SNe}$ that occur at low densities, the stronger the outflow rate is (cf. Fig. 14). SNe occurring at high densities are 'futile' events that show no correlation to the outflow rate. Photoionization can have both positive and negative effect on the outflow rate, as it not only reduces $n_{\text {SNII }}$ (positive) but also reduces the amount of star-forming gas and thus the SFR (negative). Adopting a too short stellar lifetime (3 Myr) suppresses outflows, as it gives $\mathrm{SN}$ progenitors too little time to escape from their parent clouds.

the ratio of the SFR to the outflow rate, as a function of time in the same set of runs.

Run $P E-P I-S N$ has a higher outflow rate than run $P E-n o P I-S N$, which suggests that photoionization can help drive outflows by reducing the ambient density at the locations where SNe will later explode (see equation 10 in Naab \& Ostriker 2017). This can be seen in Fig. 15, which shows a histogram of the ambient number density where the SNe have gone off $\left(n_{\mathrm{SNII}}\right)$ before $t=800 \mathrm{Myr}$ in the same set of runs.

Turning off PE heating (noPE-PI-SN and noPE-noPI-SN) increases the outflow rate because of the increased SFR. The gas in the cold phase becomes colder without the PE heating (cf. Fig. 9) which makes the gas more gravitationally unstable. It is therefore easier for it to collapse to high densities, resulting in more star-forming gas (cf. Fig. 8, bottom panel). The effect of photoionization in the two noPE runs turns out to be the opposite here, where noPE-noPI$S N$ has a slightly higher outflow rate. This is because not only does photoionization reduce $n_{\mathrm{SNII}}$, which promotes outflows, it can also suppress star formation and therefore suppress outflows. The net effect of photoionization on the outflow rate therefore depends on the competition between these two factors.

Run PE-PI-SN-3Myr has the lowest mass-loading factor, with an average value of 0.1 . Compared to run PE-PI-SN, which has a mass-loading factor $\sim 1$, it demonstrates that the adopted stellar lifetime can have a profound effect on the outflows, even though it seems to only have a very weak effect on the SFR (cf. Fig. 6). This is because $3 \mathrm{Myr}$ is much shorter than the lifetime of the majority of the massive stars, some of which can have lifetimes as long as $30 \mathrm{Myr}$. Adopting an artificially short stellar lifetime has two consequences. First, photoionization will have less time to operate and thus will be less efficient at reducing $n_{\mathrm{SNII}}$. However, the largest ionizing 

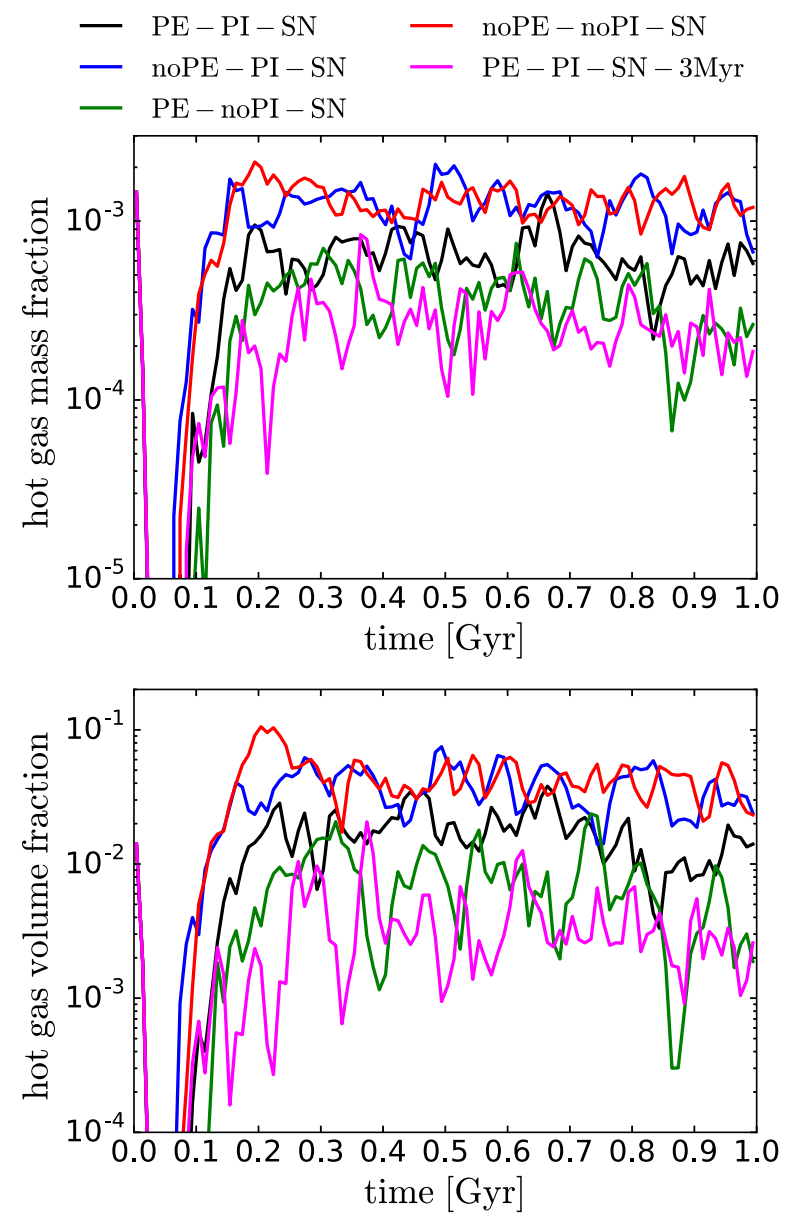

Figure 16. Time evolution of the hot gas fraction in mass (upper panel) and in volume (lower panel) in the region of $R<1.5 \mathrm{kpc}$ and $|z|<0.2 \mathrm{kpc}$. The outflow rate (cf. Fig. 14) increases with the hot gas fraction, as the outflows are driven by the overpressurized hot gas.

photon luminosities come from the most massive stars, which have lifetimes comparable to $3 \mathrm{Myr}$, and so it is unclear how important this effect is in practice. Secondly, and perhaps more importantly, the $\mathrm{SN}$ progenitors that have small ionizing photon luminosities will have less time to drift away from their parental clouds. As a result, these SNe will mostly explode within high-density environments.

It is worth noting that the ranking in outflow rate (Fig. 14) among different runs is the same as that in Fig. 15 in the low-density regime $\left(n_{\mathrm{SNII}} \lesssim 0.1 \mathrm{~cm}^{-3}\right)$. On the other hand, SNe going off in high-density environments seem to have little impact on the outflow rate. They can be viewed as 'futile events', as most of their injected energy will be radiated away rapidly. Photoionization turns most of the futile SN events into 'efficient' events, but it does not necessarily guarantee higher outflow rate as it can also reduce the total number of SN events by suppressing star formation. Eventually, it is the number of efficient SN events (instead of the fraction of the total $\mathrm{SN}$ events that are efficient) that controls the outflow rate.

Fig. 16 shows the time evolution of the hot gas $\left(T>3 \times 10^{4} \mathrm{~K}\right)$ fraction both in mass (upper panel) and in volume (lower panel) in the region of $R<1.5 \mathrm{kpc}$ and $|z|<0.2 \mathrm{kpc}$. The ranking of different runs is the same as that in the outflow rates (Fig. 14) and the number of low- $n_{\text {SNII }}$ SN events (Fig. 15). The implication is clear: when there are more $\mathrm{SNe}$ occurring in low-density environments, either because of photoionization prior to the $\mathrm{SN}$ events or because the progenitor stars drift away from their parental clouds, these SN remnants can retain their thermal energy for a longer time (as the radiative cooling rate scales with the square of the density), leading to a higher hot gas fraction that provides the necessary pressure force to drive the outflows. This picture is also qualitatively consistent with the gas morphology shown in Figs 3 and 4: PE-PI-SN-3Myr shows the smallest bubbles, and it has the lowest hot gas fraction, the fewest efficient $\mathrm{SN}$ events, and the lowest outflow rate.

\section{DISCUSSION}

\subsection{The 'primary' feedback process}

Given the different combinations of feedback we have explored, it is tempting to single out a feedback process that is the 'most important'. This may seem to be impractical due to the potential non-linear coupling among different feedback processes (Hopkins et al. 2014). Nevertheless, it is still interesting to ask which process by itself can lead to a galaxy closest to the full-feedback model. Because of the simplistic treatment we adopt for photoionization, we will just focus on PE heating and SN feedback.

Judging from the total SFR (Figs 6 and 7), at low enough surface densities (as in our fiducial disk), PE heating alone suppresses star formation as effectively as $\mathrm{SNe}$ alone can do (comparing $P E-n o P I-n o S N$ and $n o P E-n o P I-S N$ ). It is therefore insufficient to assess the relative importance of these two processes in suppressing star formation merely based on the total SFR.

On the other hand, the gas morphology and the phase diagram depend much more crucially on SNe than on PE heating (Figs 3, 4 and 9). If we assume that the full-feedback run $P E-P I-S N$ gives the most 'correct' solution, then one can still obtain gas morphologies and phase distributions with SNe alone (noPE-noPI-SN) that are close to the correct ones. This is because the SNe provide much more heating in the diffuse phase than the PE heating. Though one does end up with slightly more cold and dense gas due to the lack of PE heating, the way star formation is suppressed is qualitatively the same: SNe regulate the amount of cold and dense gas that can form out of the warm and diffuse gas reservoir.

However, the morphology and phase diagram with PE heating alone $(P E-n o P I-n o S N)$ differ substantially from the other runs. For the dense gas, PE heating is a mild process that only heats up the gas to a few hundred Kelvin at most and thus cannot inject enough energy and momentum into the ISM to disperse the clouds or drive turbulence. As such, the majority of gas remains cold and simply forms dense gas clumps due to gravity. When the gas surface density is low, these clumps can reach hydrostatic equilibrium and stop further collapse due to the locally enhanced PE heating. The way star formation is suppressed here is therefore different even qualitatively from that in the SNe runs. Moreover, this PE heating is artificially boosted, as in the 'real' ISM $(P E-P I-S N)$ the gas is on average much farther away from the stars (see Fig. 10). This is a perfect example of how different feedback processes couple with each other non-linearly.

It is worth noting that the effect of $\mathrm{SNe}$ (or any other feedback processes), when operating alone, could also be artificially enhanced, though to a lesser extent as we argue above. An additional and perhaps more compelling piece of evidence indicating the dominant importance of $\mathrm{SNe}$ in regulating star formation comes from the fact that the majority of gas in $P E-P I-S N$ is much warmer than its thermal equilibrium temperature (as shown in the one-zone model in Fig. 1), which suggests that SN heating dominates over PE heating.

Finally, we note that the SFR in the PE-only model shows a secular increase. We show in Appendix B2 that its SFR becomes 
higher than the SN-only model by a factor of two by the time $t=$ 2 Gyr, and it shows no sign of reaching a steady state. We expect that its SFR will keep increasing and may even reach a level that is too high compared to the observed dwarf galaxies if we extended the run further. ${ }^{14}$

These considerations, together with the fact that the PE-only run does not drive outflows at all, lead us to conclude that SN feedback is the primary process responsible for shaping the ISM and regulating star formation in dwarf galaxies, with PE heating playing a secondary (but still important) role.

\subsubsection{Comparison with Forbes et al. (2016)}

Our results are in conflict with those obtained by Forbes et al. (2016), who also studied the relative importance of SN feedback and PE heating for regulating star formation in a low metallicity, low surface density dwarf galaxy. Forbes et al. (2016) carried out their simulations using the ENZo hydrodynamical code (Bryan et al. 2014) and used a modified version of the GRACKLE cooling and chemistry package (Smith et al. 2017) to model the thermal evolution of the ISM. They found, using a similar setup to that studied in this paper, that $\mathrm{SNe}$ alone were ineffective at suppressing star formation, but that if they also included PE heating by a spatially and temporally varying ISRF, the resulting SFRs were in good agreement with observation. They also found that they obtained very similar SFRs in runs with only PE heating to those in runs with both PE heating and SN feedback, leading them to conclude that PE heating was the dominant feedback process. Finally, they found a large amount of cold gas in all of their runs, while in our runs, the ISM is always dominated by warm gas whenever $\mathrm{SNe}$ are included.

In our present study, we have adopted a self-consistent treatment of a spatially and temporally varying ISRF very similar to that used in Forbes et al. (2016), so it seems unlikely that our treatment of the PE heating is responsible for the tension in our results. Instead, we believe that the primary cause of this tension is an inconsistency in the Forbes et al. thermal model. Forbes et al. (2016) model the effects of metal heating and cooling in the ISM using a cooling table derived from the CLOUDY photodissociation region code (Ferland et al. 2013). Specifically, they use a table that was derived using the method of Smith, Sigurdsson \& Abel (2008) and that assumes an incident UV radiation field given by the $z=0$ model from Haardt \& Madau (2012). ${ }^{15}$ However, in an attempt to account for the effects of atomic hydrogen self-shielding in dense gas, they modified the way that this table was used within GRACKLE. ${ }^{16}$ They switched off the heating term in gas denser than $n_{\text {gas }}>0.01 \mathrm{~cm}^{-3}$, reasoning that this gas should be self-shielded and hence should not be strongly heated by the extragalactic UV background (Forbes, private communication).

Unfortunately, doing this without modifying the cooling rates leads to an inconsistency. The cooling rate in gas with $T<10^{4} \mathrm{~K}$ is highly sensitive to the fractional ionization (see e.g. fig. 2 in Dalgarno \& McCray 1972), both because the fine structure cooling rates are sensitive to the free electron number density, and also

\footnotetext{
14 On the other hand, on cosmological time-scales, our isolated galaxy setup would be missing other important processes such as gas accretion and so is not an ideal method to investigate the long-term evolution of the galaxies.

${ }^{15}$ This is the file CloudyData_UVB=HM2012.h5 in the GRACKLE package.

${ }^{16}$ See specifically change set 3 a58638 in their fork of the GRACKLE codebase, available at https://bitbucket.org/jforbes/grackle.
}

because hydrogen recombination cooling can be an important process in this regime. The cooling rates computed using CLOUDY and tabulated in the file CloudyData_UVB=HM2012.h5 were computed under the assumption that the gas was optically thin to the Haardt \& Madau (2012) UV background, i.e. they do not account for the effects of self-shielding. Therefore, the modified version of GRACKLE used by Forbes et al. (2016) calculates the heating rate of $n_{\text {gas }}>0.01 \mathrm{~cm}^{-3}$ gas under the assumption that it is self-shielded but calculates the cooling rate for the same gas under the assumption that it is optically thin, which is inconsistent. The consequence of this is that in gas with $n_{\text {gas }}>0.01 \mathrm{~cm}^{-3}$, the calculations carried out by Forbes et al. (2016) implicitly assume a fractional ionization that is much larger than it should be in self-shielded gas, and hence substantially overestimate the cooling rate, by an order of magnitude or more. This overestimated cooling rate leads to the production of large quantities of cold gas in their simulations, and consequently to an extremely large SFR in the absence of feedback. Because the gas cools so quickly, SN feedback is unable to strongly suppress this rapid star formation (primarily because it acts too late), and so a reasonable SFR is obtained only once one includes the effects of PE heating.

Simulations using a similar setup to that in Forbes et al. (2016) but that correct the cooling inconsistency (by switching to a cooling table appropriate for the case of a zero UV background in gas with $n_{\text {gas }}>0.01 \mathrm{~cm}^{-3}$ ) amongst other changes (such as introducing some initial turbulence to prevent overrapid settling of the gas disc and adopting a smaller gas scalelength) find results that differ significantly from those in the previous simulations (Forbes, private communication). The large amounts of cold, low-density gas vanish and the ISM becomes dominated by warm gas, in good agreement with our results. In addition, the SFR in the absence of feedback decreases significantly, as does the SFR with only SN feedback. Forbes et al. continue to find that runs with PE heating alone or with PE heating plus $\mathrm{SN}$ feedback are more effective at suppressing the SFR than pure SN feedback (i.e. the results of Forbes et al. 2016 remain qualitatively unchanged), but the difference in the SFR between the $\mathrm{SNe}$ only and $\mathrm{SNe}+\mathrm{PE}$ runs is now only a factor of two to three, rather than the factor of 20 found in the previous simulations (Forbes, private communication). This significantly alleviates the tension with our results. However, it does not eliminate it entirely: we find that the SFR is roughly comparable in runs with $\mathrm{SNe}$ but without PE heating and with PE heating but without $\mathrm{SNe}$ (runs noPE-PI-SN and PE-noPI-noSN; see Fig. 6) whereas Forbes et al. continue to find more effective suppression of star formation in their PE-only model than in their SN-only model. ${ }^{17}$ However, as we argue above, this does not necessarily mean that PE heating is more effective than $\mathrm{SNe}$ in dwarf galaxies as the former can be artificially boosted in a PE-only run.

Finally, we caution that there are some other potential issues in Forbes et al. (2016) which may affect their conclusions. First of all, their fiducial disc has a very low gas surface density (only $0.5 \mathrm{M}_{\odot} \mathrm{pc}^{-2}$ in the central region), which is much lower than the observed dwarf galaxies on the Kennicutt-Schmidt plane. This makes the disc very stable against gravitational collapse and

\footnotetext{
${ }^{17}$ It should be noted that the 'SN-only' model in Forbes et al. (2016) actually includes $\mathrm{SNe}$, photoionization and stellar winds from massive stars, with these three processes always being switched on and off together. For this reason, our noPE-PI-SN run is the best analogue of their SN-only run. We do not include feedback from stellar winds in our model, but we expect its effect should be sub-dominant at the metallicity we adopt (see Section 4.2.3).
} 
suppressing star formation becomes much easier, even at a very low mean temperature. In addition, due to the cooling issue discussed above, the disc vertically collapses into a very thin layer and seems to be numerically supported by their coarse spatial resolution $(2.5 \mathrm{pc})$. Moreover, their simulations are merely run for a very short amount of time $(\sim 150 \mathrm{Myr})$. Our results suggest that this is too short for the system to settle to a steady state. Lastly, the densitytemperature diagrams look puzzling: besides the overabundant cold gas as discussed above, there is a very tight 'line' in the diffuse and hot $\left(T>10^{4} \mathrm{~K}\right)$ phase even in their no-feedback model, and there is an empty region between $10 \mathrm{~K}<T<1000 \mathrm{~K}$ in particular in the no-feedback model and the PE-only model. This suggests that there may be non-trivial numerical artefacts that appear in their results.

\subsection{Potential caveats}

\subsubsection{Cosmic ray ionization}

For simplicity and to aid comparison with Forbes et al. (2016), we have deliberately neglected the heating from CR ionization which was included in Paper I. In low-metallicity systems, CR ionization heating is less important in the dense phase of the ISM compared to PE heating, while it can dominate over PE heating in the diffuse phase (see Paper I). However, as shown in Paper I, SN feedback still dominates when a constant CR ionization rate $\xi=3 \times 10^{-18} \mathrm{~s}^{-1}$ is adopted, where $\xi$ is scaled with the SFR surface density according to the Milky Way value. Therefore, we do not expect our results to change dramatically if $\mathrm{CR}$ ionization is included with a reasonable choice of $\xi$.

It is desirable to model the CR ionization heating self-consistently in a similar spirit to our treatment of PE heating. However, this is much more challenging owing to the complicated behaviour of $\mathrm{CR}$ propagation which depends on the configuration of the magnetic fields and some uncertainties in its propagation mechanisms (Uhlig et al. 2012; Hanasz et al. 2013; Girichidis et al. 2016a; Simpson et al. 2016). A fully self-consistent treatment of the CR ionization heating therefore requires substantial further investigation.

\subsubsection{Treatment of photoionization}

As mentioned in Section 2.5.2, our treatment of photoionization suffers from mass biasing that preferentially ionizes mass concentrations as it has no angular resolution. Therefore, we expect it to overreduce the amount of high-density gas and thus oversuppress star formation and overpromote galactic outflows. On the other hand, photons that escape through the directions of low column densities can in principle also suppress star formation, which our approach would fail to capture. Therefore, our approach could conceivably underestimate the effect of photoionization. However, we expect that the net effect is an overestimation as ionizing all the nearby dense gas clumps is directly relevant for suppressing star formation, while the escaped photons are not necessarily useful for suppressing star formation. Since in our simulations the SFR is insensitive to the inclusion of photoionization (see Fig. 6), the overestimation should be rather small. However, the outflow rate does show notable dependence on photoionization (e.g. compare $P E-P I-S N$ and $P E-n o P I-S N$ in Fig. 14), which can potentially be of numerical origin due to our simplistic treatment. The amount of overestimation will depend crucially on the density structure in the vicinity of photoionizing massive stars. High-resolution simulations coupled with radiative transfer will provide important information on the credibility of this simplified but cost-efficient approximation.

\subsubsection{Stellar winds}

Another important feedback mechanism we have ignored is the stellar winds from massive stars, which can play a similar role as photoionization in the sense that they both can create favourable low-density environments for the subsequent $\mathrm{SNe}$ to be efficient and thus promote outflows (Gatto et al. 2017; Peters et al. 2017). We have neglected them in this study for two reasons, one numerical and one physical. Numerically, the issue is that it is difficult to properly model stellar winds using SPH owing to the fixed SPH mass resolution. This limits the ability of SPH to capture the low but continuous mass-loss rate from massive stars, unless the mass resolution is very much better than in our whole galaxy simulations (see e.g. Dale et al. 2013). In other words, it is much more difficult to obtain a resolved and converged evolution of stellar winds using SPH as opposed to in an Eulerian grid-based code that in principle has infinite mass resolution (albeit coupled with finite spatial resolution).

Physically, the justification for ignoring stellar winds in lowmetallicity dwarf galaxies stems from the fact that the mass-loss rates of OB stars and Wolf-Rayet stars are highly sensitive to metallicity (Puls, Springmann \& Lennon 2000; Vink \& de Koter 2005). Reducing the metallicity reduces the radiative forcing and hence greatly reduces the strength of the winds. Consequently, at the metallicity examined in this study $\left(Z=0.1 \mathrm{Z}_{\odot}\right)$, stellar winds will be much less effective and much less energetic than in the local ISM. We therefore do not expect them to be a significant source of feedback in comparison to photoionization, justifying our decision to ignore them.

\section{SUMMARY}

We present high-resolution $\left(m_{\text {gas }}=4 \mathrm{M}_{\odot}, N_{\text {ngb }}=100\right)$ simulations of isolated gas-rich dwarf galaxies which include self-gravity, nonequilibrium cooling and chemistry, star formation, stellar feedback and metal enrichment. Each simulation is run for 1 Gyr. We introduce a flexible method to sample the IMF which allows for the formation of individual massive stars at our resolution. The stellar feedback includes PE heating, photoionization and SNe. Instead of assuming a constant value of $G_{0}$ throughout the disc, we selfconsistently calculate $G_{0}$ from the star particles which makes it vary both spatially and temporally. Photoionization is treated with a Strömgren-volume approach that can account for overlapping $\mathrm{H}$ II regions. In a uniform medium, the dynamical evolution of the $\mathrm{H}$ II regions converges with increasing resolution, and agrees well with that predicted by more sophisticated radiative transfer methods (Fig. A1), though in an inhomogeneous medium the method becomes strongly mass-biased. We run simulations with two initial conditions that only differ in the scalelength of the gas disc and therefore the central gas surface density $\left(\Sigma_{\text {gas }}\right)$.

We find that $\mathrm{SNe}$ play a dominant role in suppressing star formation and shaping the ISM in dwarf galaxies. The run with $\mathrm{SNe}$ alone is able to reproduce most of the ISM properties seen in the full-feedback run, such as the SFR (Fig. 6), gas morphology (Figs 3 and 4), gas distribution in the phase diagram (Fig. 11) and the galactic outflow rate (Fig. 14). Because of SNe, the majority of gas (the diffuse phase) becomes much warmer than it should be if it were in thermal equilibrium. In other words, the dynamical effect of $\mathrm{SNe}$ results in a new thermal-equilibrium relation, which makes the gas lie above the equilibrium curves in the one-zone model as shown in Fig. 1 where PE heating is the main heat source. This demonstrates that SN heating largely dominates over PE heating in the ISM of 
dwarf galaxies, and star formation is regulated by $\mathrm{SNe}$ which control the warm-to-cold transition of gas. However, as far as chemistry is concerned, $\mathrm{SNe}$ are not an important destruction mechanism of $\mathrm{H}_{2}$ compared to photodissociation, as the $\mathrm{SN}$-only run is not able to suppress the $\mathrm{H}_{2}$ fraction to the same level as the full-feedback run (Fig. 8).

On the other hand, the run with PE heating alone, while being able to suppress star formation to a similar level as the full-feedback run, generates drastically different results for all other physical properties. More importantly, the effect of PE heating is artificially boosted in the PE-only run where the dense clouds are always located very close to the UV-emitting stars due to gravity and the lack of a strong feedback mechanism to disperse the clouds (Fig. 10). As such, the mechanism that suppresses star formation in the PE-only run does not exist in the realistic ISM of dwarf galaxies, as it is not possible to have so much gas located so close to the UV sources without being dispersed by other feedback processes (such as $\mathrm{SNe}$ or photoionization). This is a good example of the non-linear coupling between different feedback processes. The artificially boosted ISRF also leads to a significantly enhanced dust continuum IR emission (by an order of magnitude) and therefore an FIR line-to-continuum ratio too low compared to the observed nearby dwarf galaxies (Fig. 12). Including SNe leads to an FIR line-to-continuum ratio of the simulated galaxies that agrees well with observations by pushing gas further away from the massive stars.

That said, the PE heating still has a notable (though secondary) effect on the ISM. PE heating reduces the amount of cold and dense gas and its inclusion in the simulation reduces the SFR by around a factor of two compared to the result in a simulation with $\mathrm{SNe}$ but without PE heating. In addition, PE heating indirectly reduces the outflow rate by reducing the $\mathrm{SN}$ rate and the hot gas fraction.

In our compact disc setup, neither SNe nor PE heating is able to suppress star formation by itself at a rate similar to that found in the full-feedback run (Fig. 6). Including photoionization is crucial in this case, which highlights the importance of pre-SN feedback from massive stars when the gas surface density becomes higher.

Photoionization can also affect the ISM in a similar way as PE heating by reducing the amount of star-forming gas and therefore the SFR. However, it does not necessarily reduce the outflow rate. This is because unlike PE heating that can only mildly heat up the dense gas (to a few hundred of Kelvin at most, see Fig. 1) and make it gravitational stable, photoionization heats the dense gas to a much higher temperature $\left(10^{4} \mathrm{~K}\right)$ which can create expanding bubbles due to the large pressure imbalance. Therefore, it can prepare a suitable low-density environment for the subsequent SNe to be efficient and therefore promote outflows. The net effect of photoionization on the outflows can thus be either positive or negative, depending on the competition between the two processes.

We find clear evidence that the galactic outflow rate is dictated by the number of SNe that occur in low-density environments (Figs 14 and 15), while the number of SNe that occur in dense environments shows little correlation with the outflow rate (see also Naab $\&$ Ostriker 2017, equation 10). The outflow rate also positively correlates with the hot gas fraction in the ISM (Fig. 16), as it is the overpressurized hot gas that drives the outflows. Our result is in very good agreement with recent studies such as Walch et al. (2015), Girichidis et al. (2016b), Gatto et al. (2017) or Peters et al. (2017). In addition to the effect of photoionization, we find that the adopted stellar lifetime also has an important effect on $n_{\text {SNII }}$. Adopting a short stellar lifetime ( $3 \mathrm{Myr}$ for all massive stars) reduces the time available for the $\mathrm{SN}$ progenitors to escape from their birth clouds, which increases $n_{\mathrm{SNII}}$ and reduces the outflow rate.

\section{ACKNOWLEDGEMENTS}

We thank the referee for the insightful and constructive comments. We thank John Forbes for valuable comments on the manuscript, and we thank Bruce Elmegreen, Sambit Roychowdhury and Diane Cormier for providing the observational data. The Center for Computational Astrophysics is supported by the Simons Foundation. This research was supported by the German Federal Ministry of Education and Research (BMBF) within the German-South-African collaboration project 01DG15006 'Ein kosmologische Modell für die Entwicklung der Gasverteilung in Galaxien'. CYH, TN, SCOG and SW acknowledge support from the Deutsche Forschungsgemeinschaft (DFG) priority program SPP 1573 'Physics of the Interstellar Medium'. SCOG acknowledges additional support from the DFG via SFB 881, 'The Milky Way System' (sub-projects B1, B2 and B8), and from the European Research Council under the European Community's Seventh Framework Programme (FP7/20072013) via the ERC Advanced Grant STARLIGHT (project number 339177). TN acknowledges support from the Cluster of Excellence 'Origin and structure of the Universe'. PCC acknowledges support from the Science and Technology Facilities Council (under grant ST/N00706/1) and the European Communityś Horizon 2020 Programme H2020-COMPET-2015, through the StarFormMapper project (number 687528). SW additionally thanks the DFG for support through SFB 956, 'Conditions and impact of star formation' (sub-project C5), and the European Research Council under the European Communityś Eighth Framework Programme FP8 via the ERC-Starting-Grant RADFEEDBACK (proposal no. 679852).

\section{REFERENCES}

Bakes E. L. O., Tielens A. G. G. M., 1994, ApJ, 427, 822

Bate M. R., Burkert A., 1997, MNRAS, 288, 1060

Bate M. R., Bonnell I. A., Price N. M., 1995, MNRAS, 277, 362

Begum A., Chengalur J. N., Karachentsev I. D., Sharina M. E., Kaisin S. S., 2008, MNRAS, 386, 1667

Bergin E. A., Hartmann L. W., Raymond J. C., Ballesteros-Paredes J., 2004, ApJ, 612, 921

Bigiel F., Leroy A., Walter F., Blitz L., Brinks E., de Blok W. J. G., Madore B., 2010, AJ, 140, 1194

Bisbas T. G. et al., 2015, MNRAS, 453, 1324

Bryan G. L. et al., 2014, ApJS, 211, 19

Cen R., Ostriker J. P., 1992, ApJ, 399, L113

Chieffi A., Limongi M., 2004, ApJ, 608, 405

Cigan P. et al., 2016, AJ, 151, 14

Clark P. C., Glover S. C. O., Klessen R. S., 2012, MNRAS, 420, 745

Cormier D. et al., 2015, A\&A, 578, A53

Cullen L., Dehnen W., 2010, MNRAS, 408, 669

da Silva R. L., Fumagalli M., Krumholz M., 2012, ApJ, 745, 145

da Silva R. L., Fumagalli M., Krumholz M. R., 2014, MNRAS, 444, 3275

Dale J. E., Bonnell I., 2011, MNRAS, 414, 321

Dale J. E., Ngoumou J., Ercolano B., Bonnell I. A., 2013, MNRAS, 436, 3430

Dalgarno A., McCray R. A., 1972, ARA\&A, 10, 375

Dalla Vecchia C., Schaye J., 2012, MNRAS, 426, 140

Dehnen W., Aly H., 2012, MNRAS, 425, 1068

Dekel A., Silk J., 1986, ApJ, 303, 39

Draine B. T., 1978, ApJS, 36, 595

Elmegreen B. G., Hunter D. A., 2015, ApJ, 805, 145

Federrath C., Banerjee R., Clark P. C., Klessen R. S., 2010, ApJ, 713, 269

Ferland G. J. et al., 2013, Rev. Mex. Astron. Astrofis., 49, 137

Forbes J. C., Krumholz M. R., Goldbaum N. J., Dekel A., 2016, Nature, 535,523

Gatto A. et al., 2017, MNRAS, 466, 1903

Georgy C. et al., 2013, A\&A, 558, A103 
Gingold R. A., Monaghan J. J., 1977, MNRAS, 181, 375

Girichidis P. et al., 2016a, ApJ, 816, L19

Girichidis P. et al., 2016b, MNRAS, 456, 3432

Glover S. C. O., 2003, ApJ, 584, 331

Glover S. C. O., Clark P. C., 2012a, MNRAS, 421, 116

Glover S. C. O., Clark P. C., 2012b, MNRAS, 426, 377

Glover S. C. O., Mac Low M.-M., 2007, ApJS, 169, 239

Glover S. C. O., Smith R. J., 2016, MNRAS, 462, 3011

Haardt F., Madau P., 2012, ApJ, 746, 125

Habing H. J., 1968, Bull. Astron. Inst. Neth., 19, 421

Hanasz M., Lesch H., Naab T., Gawryszczak A., Kowalik K., Wóltański D., 2013, ApJ, 777, L38

Hill A. S., Joung M. R., Mac Low M.-M., Benjamin R. A., Haffner L. M., Klingenberg C., Waagan K., 2012, ApJ, 750, 104

Hopkins P. F., 2013, MNRAS, 428, 2840

Hopkins P. F., Quataert E., Murray N., 2012, MNRAS, 421, 3488

Hopkins P. F., Kereš D., Oñorbe J., Faucher-Giguère C.-A., Quataert E., Murray N., Bullock J. S., 2014, MNRAS, 445, 581

Hubber D. A., Walch S., Whitworth A. P., 2013, MNRAS, 430, 3261

Hu C.-Y., Naab T., Walch S., Moster B. P., Oser L., 2014, MNRAS, 443, 1173

Hu C.-Y., Naab T., Walch S., Glover S. C. O., Clark P. C., 2016, MNRAS, 458,3528

Hunter D. A. et al., 2012, AJ, 144, 134

Janka H.-T., 2012, Annu. Rev. Nucl. Part. Sci., 62, 407

Joung M. K. R., Mac Low M.-M., 2006, ApJ, 653, 1266

Katz N., 1992, ApJ, 391, 502

Kennicutt R. C., Jr, 1998, ApJ, 498, 541

Kim C.-G., Kim W.-T., Ostriker E. C., 2011, ApJ, 743, 25

Kim J.-h., Krumholz M. R., Wise J. H., Turk M. J., Goldbaum N. J., Abel T., 2013a, ApJ, 775, 109

Kim J.-h., Krumholz M. R., Wise J. H., Turk M. J., Goldbaum N. J., Abel T., 2013b, ApJ, 779, 8

Kroupa P., 2001, MNRAS, 322, 231

Krumholz M. R., 2012, ApJ, 759, 9

Larson R. B., 1974, MNRAS, 169, 229

Lejeune T., Cuisinier F., Buser R., 1997, A\&AS, 125

Lejeune T., Cuisinier F., Buser R., 1998, A\&AS, 130, 65

Li Y., Mac Low M.-M., Klessen R. S., 2005, ApJ, 620, L19

Lucy L. B., 1977, AJ, 82, 1013

Mac Low M.-M., Ferrara A., 1999, ApJ, 513, 142

Moster B. P., Somerville R. S., Maulbetsch C., van den Bosch F. C., Macciò A. V., Naab T., Oser L., 2010, ApJ, 710, 903

Moster B. P., Naab T., White S. D. M., 2013, MNRAS, 428, 3121

Naab T., Ostriker J. P., 2017, ARA\&A, in press

Navarro J. F., Frenk C. S., White S. D. M., 1997, ApJ, 490, 493

Nelson R. P., Langer W. D., 1997, ApJ, 482, 796

Ossenkopf V., Henning T., 1994, A\&A, 291, 943

Peters T. et al., 2017, MNRAS, 466, 3293

Price D. J., 2008, J. Comput. Phys., 227, 10040

Puls J., Springmann U., Lennon M., 2000, A\&AS, 141, 23

Read J. I., Hayfield T., Agertz O., 2010, MNRAS, 405, 1513

Rosdahl J., Schaye J., Teyssier R., Agertz O., 2015, MNRAS, 451, 34

Roychowdhury S., Huang M.-L., Kauffmann G., Wang J., Chengalur J. N., 2015, MNRAS, 449, 3700

Saitoh T. R., Makino J., 2013, ApJ, 768, 44

Schmidt M., 1959, ApJ, 129, 243

Schruba A. et al., 2011, AJ, 142, 37

Simpson C. M., Pakmor R., Marinacci F., Pfrommer C., Springel V., Glover S. C. O., Clark P. C., Smith R. J., 2016, ApJ, 827, L29

Smith B., Sigurdsson S., Abel T., 2008, MNRAS, 385, 1443

Smith B. D. et al., 2017, MNRAS, 466, 2217

Somerville R. S., Davé R., 2015, ARA\&A, 53, 51

Sormani M. C., Treß R. G., Klessen R. S., Glover S. C. O., 2017, MNRAS, 466, 407

Spitzer L., 1978, Physical Processes in the Interstellar Medium. Wiley, New York

Springel V., 2005, MNRAS, 364, 1105
Springel V., Di Matteo T., Hernquist L., 2005, MNRAS, 361, 776

Teich Y. G. et al., 2016, ApJ, 832, 85

Truelove J. K., Klein R. I., McKee C. F., Holliman, II J. H., Howell L. H., Greenough J. A., 1997, ApJ, 489, L179

Uhlig M., Pfrommer C., Sharma M., Nath B. B., Enßlin T. A., Springel V., 2012, MNRAS, 423, 2374

Vink J. S., de Koter A., 2005, A\&A, 442, 587

Walch S. K., Whitworth A. P., Bisbas T., Wünsch R., Hubber D., 2012, MNRAS, 427, 625

Walch S. et al., 2015, MNRAS, 454, 238

Westera P., Lejeune T., Buser R., Cuisinier F., Bruzual G., 2002, A\&A, 381 , 524

White S. D. M., Rees M. J., 1978, MNRAS, 183, 341

Wiersma R. P. C., Schaye J., Smith B. D., 2009, MNRAS, 393, 99

Wolfire M. G., McKee C. F., Hollenbach D., Tielens A. G. G. M., 2003 , ApJ, 587, 278

\section{APPENDIX A: CONVERGENCE TEST OF THE D-TYPE EXPANSION}

In this test, four star particles are placed in the centre of a uniform box that has a number density $n_{\mathrm{gas}}=100 \mathrm{~cm}^{-3}$ and gas temperature $T=10^{3} \mathrm{~K} .{ }^{18}$ The medium is composed of pure hydrogen, i.e. $X_{\mathrm{H}}=1$. Each star particle has an ionizing photon rate of $2.5 \times 10^{48} \mathrm{~s}^{-1}$ so that the total ionizing photon rate is $S_{*, \text { tot }}=10^{49} \mathrm{~s}^{-1}$, which is typical for an O star. This leads to an initial Strömgren radius $R_{\mathrm{s}}=3.1 \mathrm{pc}$.

With this setup, we expect the ionizing photons from the central sources to create an $\mathrm{H}$ II region bounded by an ionization front at position $R_{\mathrm{IF}}$. Initially, when $R_{\mathrm{IF}} \ll R_{\mathrm{s}}$, the front is R-type and expands rapidly. However, once $R_{\mathrm{IF}}$ approaches $R_{\mathrm{s}}$, the expansion velocity of the front decreases rapidly and the front undergoes a transition to a D-type front. Our photoionization algorithm does not capture the initial evolution of the R-type ionization front, since we assume that the ionization front expands to $R_{\mathrm{IF}}=R_{\mathrm{S}}$ instantaneously. However, the code should be capable of following the subsequent $\mathrm{D}$ type expansion of the front. Since this can be solved for analytically in this simple scenario, this provides a useful test of code and allows us to establish the mass resolution required to accurately follow the D-type expansion.

Fig. A1 shows the time evolution of the ionization front radius $R_{\mathrm{IF}}$, defined here as the maximum radius where a gas particle with an ionization fraction $x_{\mathrm{H}^{+}}>0.95$ can be found. The test is performed with four different resolutions with gas particle masses $m_{\mathrm{g}}=4,0.5,0.625$ and $0.0078125 \mathrm{M}_{\odot}$, respectively. The dashed line shows the classic Spitzer solution (Spitzer 1978):

$R_{\mathrm{Spi}}=R_{\mathrm{s}}\left(1+\frac{7}{4} \frac{c_{i} t}{R_{\mathrm{S}}}\right)^{4 / 7}$

where $c_{i}$ is the sound speed of the ionized medium and $t$ is the simulation time. The Spitzer solution describes the early evolution of $R_{\mathrm{IF}}$ when the pressure imbalance across $R_{\mathrm{IF}}$ is large. At a later stage, as the $\mathrm{H}_{\text {II }}$ region expands, the pressure imbalance decreases and eventually the expansion stops when it reaches pressure equilibrium, as opposed to the Spitzer solution which expands forever. This latetime evolution is investigated in Bisbas et al. (2015) using several different radiative transfer codes as part of the code comparison project STARBENCH. This study found a simple semi-empirical prescription that accurately represented the late-time behaviour of the

${ }^{18}$ This ambient temperature of $10^{3} \mathrm{~K}$ is much higher than the typical temperature in the star-forming gas which usually has $T<100 \mathrm{~K}$. The value is chosen in order to more easily compare with results obtained from radiative transfer codes in Bisbas et al. (2015) (see below). 

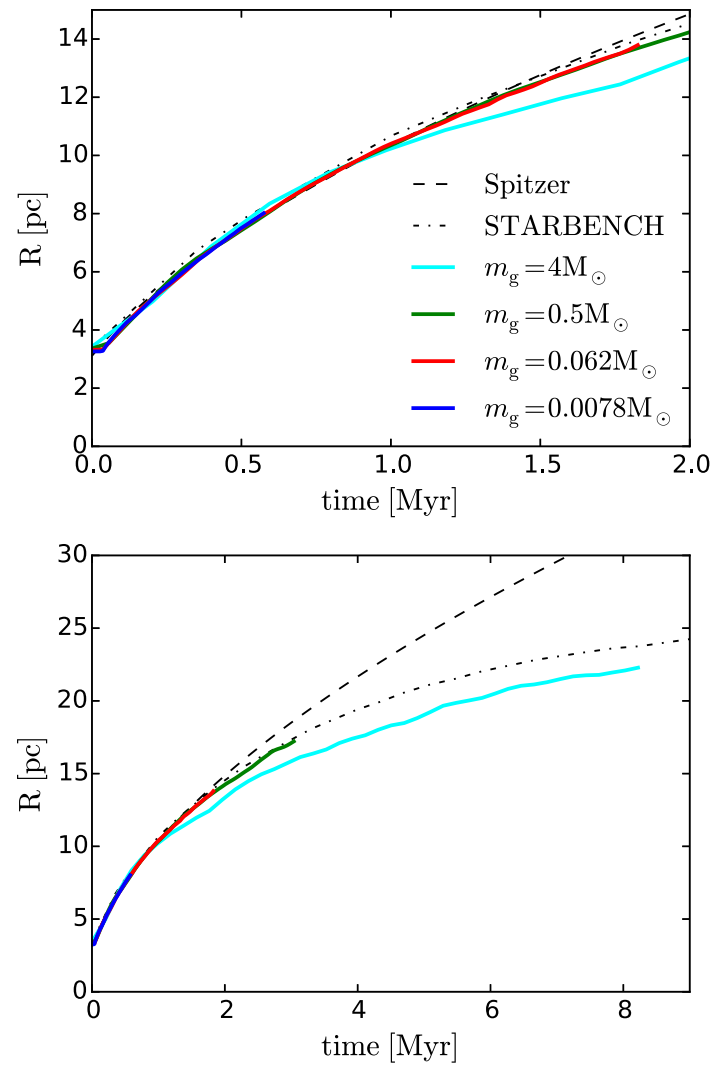

Figure A1. Time evolution of the ionization front $R_{\mathrm{IF}}$ produced by four stars with an ionizing photon rate of $2.5 \times 10^{48} \mathrm{~s}^{-1}$ each, located in the centre of a uniform box with initial density $n_{\text {gas }}=100 \mathrm{~cm}^{-3}$ and temperature $T=10^{3} \mathrm{~K}$. The convergence test is performed with four different resolutions, with gas particle masses $m_{\mathrm{g}}=4,0.5,0.625$ and $0.0078125 \mathrm{M}_{\odot}$, respectively. The dashed line shows the Spitzer solution (Spitzer 1978), which accurately describes the early-time evolution, while the dashed-dotted line shows the semi-empirical result obtained by the STARBENCH project (Bisbas et al. 2015), which accurately describes the late-time behaviour of the front. At early times, all of the runs agree well with the analytical prescription, while at late times, the results converge to the STARBENCH solution as long as $m_{\mathrm{g}} \leq$ $0.5 \mathrm{M}_{\odot}$.

D-type front, which is shown as the dashed-dotted line in Fig. A1. The upper and lower panels in the figure show the early and late evolution, respectively.

As described in Section 2.5.2, our approach is able to account for overlapping $\mathrm{H}_{\mathrm{II}}$ regions. This can be seen at $t=0$ in Fig. A1 where all four runs accurately reproduce $R_{\mathrm{S}}=3.1 \mathrm{pc}$. This is different from the non-iterative approach sometimes adopted in the literature, where overlapping $\mathrm{H}$ II regions suffer from 'double-counting'. For this test problem, this non-iterative approach would lead to four times fewer gas particles becoming ionized than should be the case. Moreover, our neighbour search is done globally instead of only on the local domain and thus is independent of domain decomposition. ${ }^{19}$

In the early stage, as expected, the Spitzer solution and the STAR$\mathrm{BENCH}$ solution agree well with each other. Our four runs show excellent converging behaviour which agrees with the analytic solution at all resolutions until $t=1 \mathrm{Myr}$, when the $m_{\mathrm{g}}=4 \mathrm{M}_{\odot}$ run

\footnotetext{
${ }^{19}$ In this test, we have run on both 16 and 32 processors in parallel and
} found essentially the same results. starts to underestimate $R_{\mathrm{IF}}$. At $t \gtrsim 2 \mathrm{Myr}$, the Spitzer solution starts to overpredict $R_{\mathrm{IF}}$ as it neglects the pressure of the ambient medium. Except for the $m_{\mathrm{g}}=4 \mathrm{M}_{\odot}$ run, the other three runs show excellent agreement with the STARBENCH solution throughout the simulation time. We conclude that with the resolution we adopted in this work ( $m_{\mathrm{g}}=4 \mathrm{M}_{\odot}$ ), we expect to slightly underestimate $R_{\mathrm{IF}}$ in a uniform medium due to insufficient resolution, although even at late times the error in $R_{\mathrm{IF}}$ remains relatively small.

\section{APPENDIX B：PARAMETER STUDY}

\section{B1 Star formation threshold}

In this section, we investigate the consequences of varying the star formation threshold $N_{\text {th }}$ (defined in Section 2.3.2). We run two additional runs that are identical to the $P E-P I-S N$ run except for the adopted $N_{\text {th }}: P E-P I-S N-M J 2$ and PE-PI-SN-MJ32 with $N_{\text {th }}=2$ and 32 , respectively; recall that in run $P E-P I-S N, N_{\text {th }}=8$. Note that a smaller $N_{\text {th }}$ corresponds to, effectively, a higher density threshold at a fixed temperature.

Fig. B1 shows the time evolution of the SFR (upper panel) and the galactic outflow rate (lower panel). Run PE-PI-SN-MJ2 begins to form stars the latest (after $t \sim 100 \mathrm{Myr}$ ) as the gas has to collapse to higher densities and lower temperatures in order to be star-forming. Besides that, no systematic difference can be found among the three runs. The SFR and the outflow rate seem to be insensitive to $N_{\text {th }}$.

Fig. B2 shows the histograms of the number density of the total gas (left-hand panel) and the star-forming gas (right-hand panel), averaged over a period from $t=400 \mathrm{Myr}$ to $t=900 \mathrm{Myr}$ with a time interval of $10 \mathrm{Myr}$. Smaller $N_{\text {th }}$ leads to more dense gas as the gas
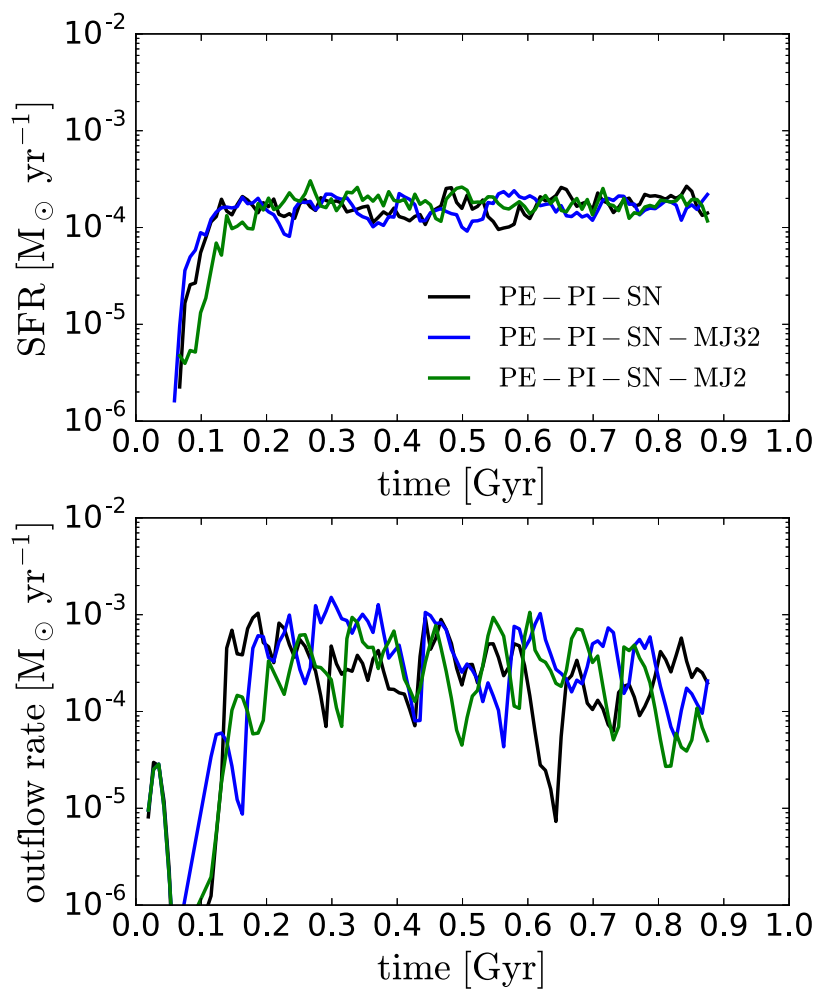

Figure B1. Time evolution of the SFR (upper panel) and the galactic outflow rate (lower panel) of the entire disk. PE-PI-SN-MJ2,PE-PI-SN, PE-PI$S N$-MJ32 adopt $N_{\text {th }}=2,8$ and 32, respectively. The SFR and the outflow rate seem to be insensitive to $N_{\text {th }}$. 


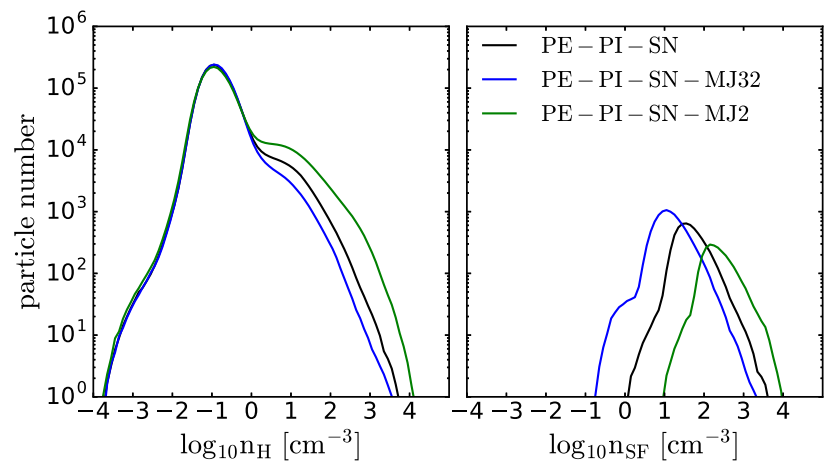

Figure B2. Histograms of the number density of the total gas (left-hand panel) and the star-forming gas (right-hand panel), averaged over a period from $t=400 \mathrm{Myr}$ to $t=900 \mathrm{Myr}$ with a time interval of $10 \mathrm{Myr}$. Smaller $N_{\text {th }}$ leads to more dense gas as the gas has to collapse to higher densities and lower temperatures in order to form stars, which delays the subsequent stellar feedback to counter further collapse. On the other hand, the low-density tail which corresponds to the hot bubble gas is insensitive to $N_{\text {th }}$.

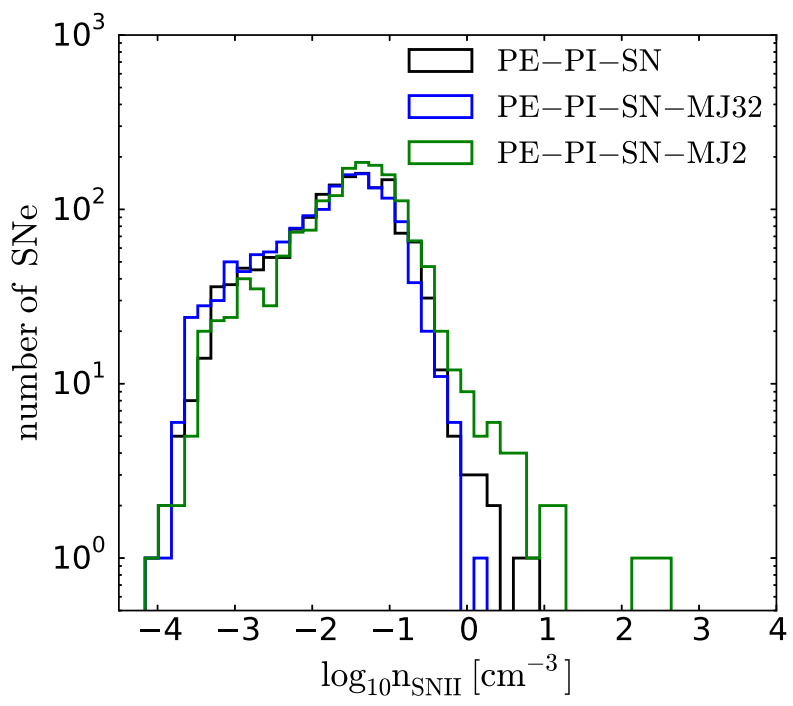

Figure B3. Histogram of the ambient hydrogen number density where the SNe occur $\left(n_{\text {SNII }}\right)$ before $t=800 \mathrm{Myr}$. Lowering $N_{\text {th }}$ increases the number of $\mathrm{SNe}$ that occur at high densities, while the number of SNe that occur at low densities remains unchanged. As a result, the outflow rate is also insensitive to $N_{\text {th }}$ (see Fig. B1).

has to collapse to higher densities and lower temperatures before it can form stars, which delays the subsequent stellar feedback to counter further collapse. The peak density of the star-forming gas also increases as $N_{\text {th }}$ decreases for the same reason. On the other hand, the low-density tail that corresponds to the hot bubble gas is insensitive to $N_{\text {th }}$

Fig. B3 shows the histogram of the ambient hydrogen number density where the SNe occur $\left(n_{\mathrm{SNII}}\right)$ before $t=800 \mathrm{Myr}$. Lowering $N_{\text {th }}$ increases the number of SNe that occur at high densities, which would not affect the outflows as these are the futile events. On the other hand, the number of $\mathrm{SNe}$ that occur at low densities is insensitive to $N_{\text {th }}$. As a result, the outflow rate is also insensitive to $N_{\text {th }}$ (see Fig. B1).

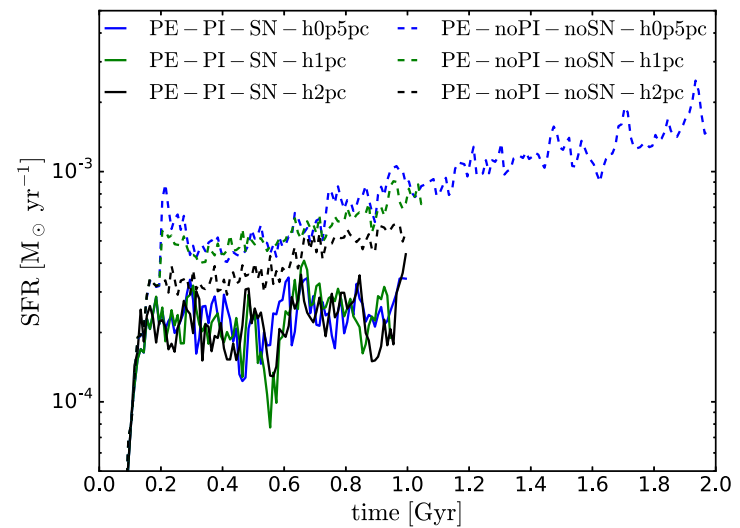

Figure B4. Time evolution of the SFR in the PE-PI-SN model (solid lines) and the PE-only model (dashed lines) with $h=2 \mathrm{pc}$ (black), $1 \mathrm{pc}$ (green) and $0.5 \mathrm{pc}$ (blue), respectively.

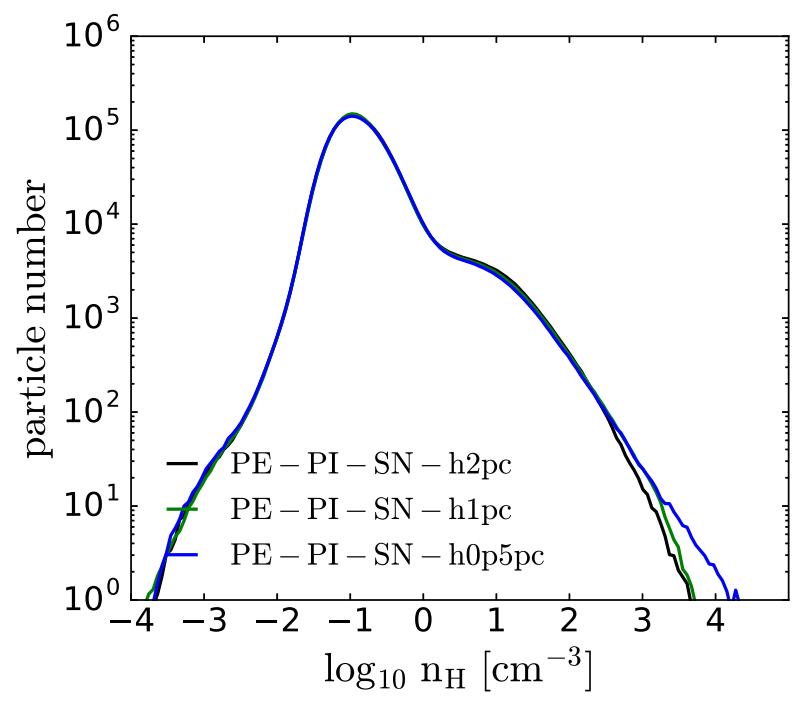

Figure B5. Histograms of the hydrogen number density in the PE-PI-SN model with the gravitational softening $h=2 \mathrm{pc}$ (black), $1 \mathrm{pc}$ (green) and $0.5 \mathrm{pc}$ (blue), respectively. According to our star formation criteria, most stars are formed around $n_{\mathrm{H}} \sim 50 \mathrm{~cm}^{-3}$ (see Fig. 9). $h$ has a negligible effect on the density distribution.

\section{B2 Gravitational softening}

In this section, we investigate how the choice of gravitational softening length $(h)$ affects our results. We rerun the simulation of the $P E-P I-S N$ and PE-noPI-noSN models (where $h=2 \mathrm{pc}$ is adopted) with $h=1$ and $0.5 \mathrm{pc}$. Simulations are restarted from $t=200 \mathrm{Myr}$. Fig. B4 shows the time evolution of the SFR in the PE-PI-SN model (solid lines) and the PE-only model (dashed lines) with $h=2 \mathrm{pc}$ (black), $1 \mathrm{pc}$ (green) and $0.5 \mathrm{pc}$ (blue), respectively. The choice of $h$ has a negligible effect on the SFR in the PE-PI-SN model where the SFR is regulated by feedback from $\mathrm{SNe}$ and photoionization. In the PE-only model, $h$ does have a slight effect (within a factor of two) on the SFR. It is interesting to note that the PE-only model shows a secular increase of SFR over time and never really reaches a steady state even up to $2 \mathrm{Gyr}$ that we explored in the run with $h=0.5 \mathrm{pc}$.

In Fig. B5 we show the histograms of the hydrogen number density in the PE-PI-SN model with different choices of $h$, which demonstrates that $h$ has little effect on the density distribution, except for the very dense gas $\left(n_{\mathrm{H}}>500 \mathrm{~cm}^{-3}\right)$ that is only a tiny 


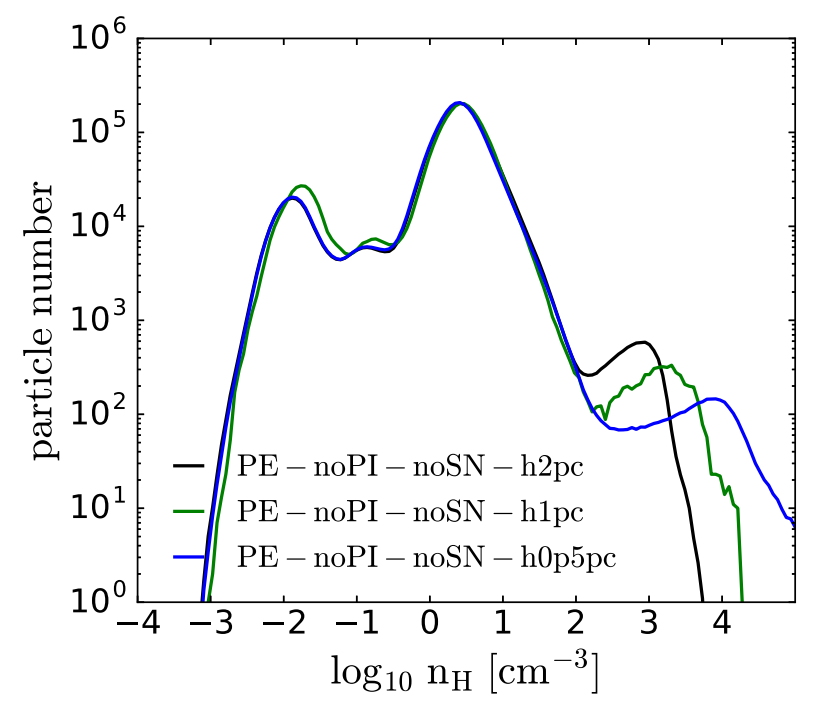

Figure B6. Same as Fig. B5 but for the PE-noPI-noSN model. The dense gas $\left(n_{\mathrm{H}}>100 \mathrm{~cm}^{-3}\right)$ becomes denser as $h$ increases.

fraction of the total star forming gas. As a comparison, according to our star formation criteria (Section 2.3.2), most stars are formed at $n_{\mathrm{H}} \sim 50 \mathrm{~cm}^{-3}$ (see Fig. 9). This explains why the SFR is insensitive to $h$ in this model (Fig. B4). On the other hand, the histograms of the hydrogen number density in the PE-only model (Fig. B6) demonstrate that the choice of $h$ can have significant effect on the high-density gas $\left(n_{\mathrm{H}}>100 \mathrm{~cm}^{-3}\right)$. The dense gas becomes denser as $h$ increases. The difference is largest in the regime where the Jeans mass is unresolved (or only marginally resolved), as expected. Fortunately, the total SFR, as shown in Fig. B4, has only a weak dependence on $h$ and therefore our main argument is unaffected.

In Fig. B7 we show the time evolution of the outflow rate (upper panel) and the mass-loading factor (lower panel) in the PE-PI-SN model with different choices of $h$. Both quantities are insensitive to $h$, and the differences between different runs are mainly originated from the time fluctuations of the outflow rate.
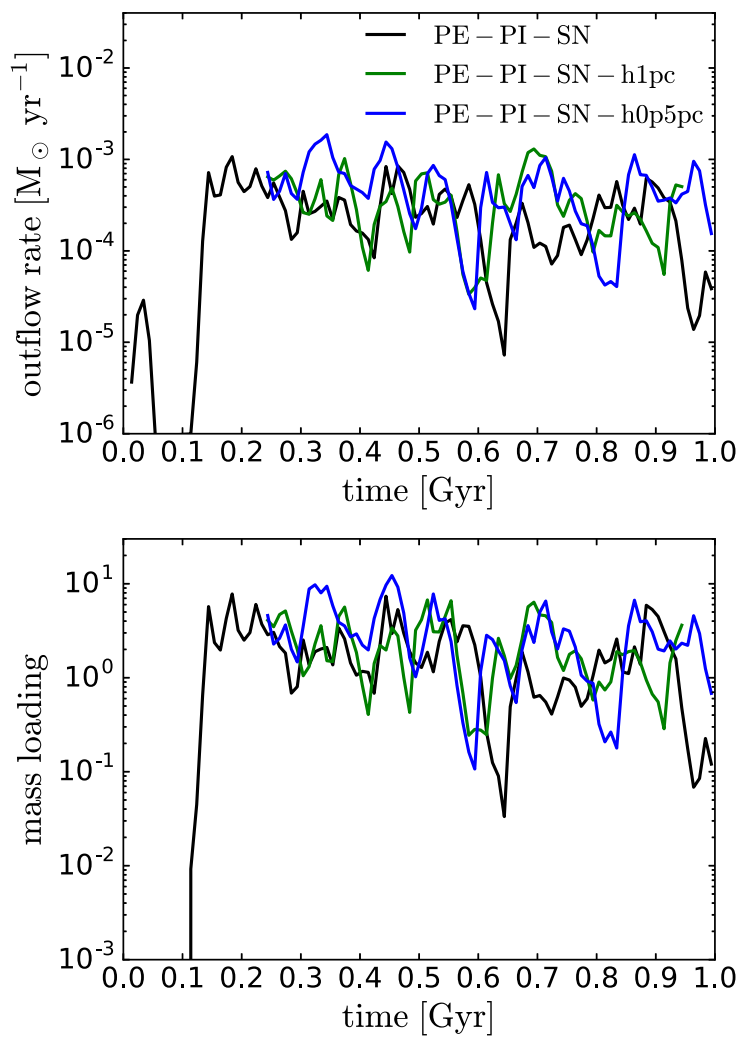

Figure B7. Time evolution of the outflow rate and the mass-loading factor in the PE-PI-SN model with $h=2 \mathrm{pc}$ (black), $1 \mathrm{pc}$ (green) and $0.5 \mathrm{pc}$ (blue), respectively. The averaged outflow rate and the mass-loading factor are not sensitive to $h$ and the differences mainly originate from the time fluctuations of the outflow rate.

This paper has been typeset from a $\mathrm{T}_{\mathrm{E}} \mathrm{X} / \mathrm{L} \mathrm{T} \mathrm{E} \mathrm{X}$ file prepared by the author. 